\title{
Thermal drag revisited: Boltzmann versus Kubo
}

\author{
Suhas Gangadharaiah and A. L. Chernyshev \\ Department of Physics, University of California, Irvine, California 92697, USA \\ Wolfram Brenig \\ Institut für Theoretische Physik, Technische Universität Braunschweig, 38106 Braunschweig, Germany
}

(Dated: October 17, 2018)

\begin{abstract}
The effect of mutual drag between phonons and spin excitations on the thermal conductivity of a quantum spin system is discussed. We derive general expression for the drag component of the thermal current using Boltzmann equation as well as Kubo linear-response formalism to leading order in the spin-phonon coupling. We demonstrate that aside from higher-order corrections which appear in the Kubo formalism both approaches yield identical result for the drag thermal conductivity. We discuss the range of applicability of our result and provide a generalization of our consideration to the cases of fermionic excitations and to anomalous forms of boson-phonon coupling. Several asymptotic regimes of our findings relevant to realistic situations are highlighted.
\end{abstract}

PACS numbers: 72.10.Bg 72.20.Pa 75.10.Jm

\section{INTRODUCTION}

Transport phenomena form a prominent group of problems in condensed matter physics. They provide a unique information on the excitations and their interactions not accessible by other methods $\stackrel{1,2}{=}$ Recently, thermal transport by spin excitations in low-dimensional quantum magnets has received significant attention due to very large heat conductivities found in a number of materials, for reviews see Refs. 3, 4. One may speculate that thermal conductivity could be used to probe elementary excitations in quantum magnets in a fashion analogous to the use of electrical conductivity in metals.

By now it is well established that integrable onedimensional quantum magnets allow for infinite heat

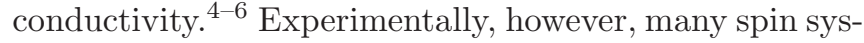
tems rather remote from integrability also demonstrate large heat conductivities $\frac{3,7}{3}$ Understanding the role of coupling of the spin degrees of freedom to an environment, such as phonons and impurities, could be essential in this context. Phonons are ubiquitous heat carriers along with spin excitations in all quantum magnets. Usually, interaction between spins and phonons is discussed in the context of dissipation of their respective currents. Significant progress has been made here,,$\underline{-10}$ yet, many questions remain open.

In this work we focus on one such question which is rarely addressed: the off-diagonal effect of the flow of one of the excitations facilitating the flow of the other $\underline{11}-13$ It is referred to as "spin-phonon drag", in analogy with electron-phonon drag discussed in the thermoelectric phenomena in metals and semiconductors $14-20$.

The second question we address in this work is the relation between two distinct theoretical approaches to transport in a generic coupled two-component system, namely the quasi-classical Boltzmann transport theory and the Kubo linear-response formalism. Such relations, while of fundamental importance, remain unclear between many techniques ${ }^{21-30}$ devised in the past. For selected prob- lems and techniques such correspondence has been estab-

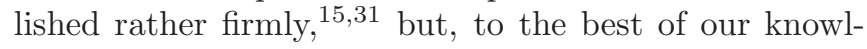
edge, the comparison discussed in this work has not been performed previously.

Historically, the term phonon drag appears in two rather distinct contexts. The first connotation is the negative effect of phonons on the electrical conductivity by slowing down electrons via processes that are different from the direct scattering effects $\underline{17,18}$ The second, also referred to as the Gurevich effect,,$\frac{119}{19}$ is responsible for the dramatic deviation of the thermopower in many materials from the predictions of the "electron-only" theory $\underline{20}$ In its idealized version, $, 1,19$ the phonon drag results in a substantial heat flow due to adjustment of the momentum distribution of phonons to that of the electrons, the latter being displaced by the electric field.

In this work, the "thermal-only" analog of the Gurevich effect for a generic spin-phonon problem is considered. In this case, only the thermal current is of interest. In the standard electron-phonon problem, the thermalonly drag is discarded traditionally. This is because the thermal conductivity by phonons in metals can usually be neglected due to strong scattering of phonons and the very large ratio of the Fermi to sound velocity $\frac{1}{1}$ However, this is not the case in several magnetic insulators of current interest $\mathrm{t}^{3.7,32-35}$ where the magnetic and lattice heat conductivity can be of the same order. Therefore, the drag between spin excitations and phonons can be an important phenomenon. We also note in passing, that in contrast the previous electron-phonon problems the dimensionality of the spin and the phonon system in magnetic insulators can be different. Spin excitations may be confined to chains, ladders, and planes. Thus, the focus of our study is on the general problem of a two-component system and the drag effect in the thermal conductivity.

While the spin-phonon problem is our main motivation, we consider a generic model of bosonic quasiparticle-like excitations, e.g. magnons, coupled to 
phonons. We derive the contribution of the phonon drag to the thermal conductivity in the lowest order of the boson-phonon coupling using Kubo and Boltzmann formalisms. We demonstrate that both approaches yield identical results for the drag component of the thermal conductivity, thus establishing a direct correspondence between these methods. We note that despite a significant body of work on thermoelectric phenomena, to the best of our knowledge, such results have not been discussed before within these two approaches.

While most of the work is devoted to bosonic spin excitations, we have also generalized our consideration to the case of fermionic excitations, as well as to the case of particle non-conserving boson-phonon interactions. The latter are as common as the "normal", particle-conserving ones and occur, e.g., in the phases with broken symmetry. We also note that using our expressions for the drag thermal conductivity we reproduce some of the results of the recent work $\frac{12}{2}$ which considers spin-phonon drag in a particular class of quantum magnets using the memoryfunction approach.

The paper is organized as follows. In Sec. [11] we introduce the model. Section III outlines the derivation of the drag conductivity from the Boltzmann equation. In Section IV we detail the Kubo diagrams for drag and elaborate on one approach to their evaluation. Sec. $\mathrm{V}$ extends the consideration of the drag thermal conductivity onto fermionic excitations as well as onto anomalous (particlenon-conserving) boson-phonon coupling. In Sec. VI we provide a qualitative discussion of thermal drag for several representative cases and asymptotic regimes. We conclude with Sec. VII Several Appendices are provided. In Appendix $\mathrm{A}$ we detail more technical points of the Boltzmann approach. Appendix $\mathrm{B}$ is devoted to the evaluation of the Kubo diagrams for the drag using an alternative approach. Finally, in Appendix $\mathrm{C}$ we discuss some corrections beyond the Boltzmann results from the Kubo diagrams.

\section{MODEL}

Spin systems can yield a wide variety of excitations, such as spinons, magnons, triplet excitations, etc., depending on the dimensionality, spin value, and geometry of the structural arrangement. Because the focus of this work is on the drag between spin excitations and phonons, we assume that both of them are describable by well-defined quasiparticles. For the spin excitations we assume energy dispersion $\varepsilon_{\mathbf{k}}$ and some phenomenological intrinsic or extrinsic transport relaxation rate. This rate may include scattering caused by impurities, spin-spin interaction, and other degrees of freedom. The corresponding energy for phonons will be denoted by $\omega_{\mathbf{p}}$ and they are also assumed to have a finite relaxation rate. In most of the paper, we assume the statistics of the spin excitations to be that of bosons. Sec. $\mathrm{V}$ outlines the changes in the drag conductivity which results if the choice of the statistics would be that of fermions.

The spin-phonon coupling occurs because lattice displacements cause changes in the spin interactions and anisotropies $\underline{36}$ Thus, the simplest, yet very general form of the spin-phonon coupling is linear in the lattice displacement and quadratic in the spin operators. After mapping spins onto bosonic quasiparticles, the resultant lowest-order boson-phonon coupling will generally contain the "normal" part, which conserves the boson number, $\underline{\underline{9}}$ and the off-diagonal, "anomalous" bosonic terms ${ }^{36}$ Since the subsequent drag conductivity derivation is conceptually identical for the normal and anomalous forms of interactions, we will postpone consideration of the latter until Sec. $\mathrm{V}$ and will treat it in less detail. Here and in the next two Sections, we will concentrate on the normal form of the coupling.

Altogether, the Hamiltonian implied for our subsequent consideration is

$$
\begin{aligned}
& H=H_{\mathrm{b}}+H_{\mathrm{ph}}+H_{\mathrm{b}-\mathrm{ph}}, \\
& H_{\mathrm{b}}=\sum_{\mathbf{k}} \varepsilon_{\mathbf{k}} b_{\mathbf{k}}^{\dagger} b_{\mathbf{k}}, \quad H_{\mathrm{ph}}=\sum_{\mathbf{p}} \omega_{\mathbf{p}} a_{\mathbf{p}}^{\dagger} a_{\mathbf{p}}, \\
& H_{\mathrm{b}-\mathrm{ph}}=\sum_{\mathbf{k}, \mathbf{p}} V_{\mathbf{p} ; \mathbf{k}, \mathbf{k}-\mathbf{p}}^{\mathrm{b}-\mathbf{p}} b_{\mathbf{k}-\mathbf{p}}^{\dagger} b_{\mathbf{k}}\left(a_{\mathbf{p}}^{\dagger}+a_{-\mathbf{p}}\right),
\end{aligned}
$$

where $b_{\mathbf{k}}^{(\dagger)}$ and $a_{\mathbf{p}}^{(\dagger)}$ are boson and phonon operators, and $V_{-\mathbf{p} ; \mathbf{k}-\mathbf{p}, \mathbf{k}}^{\mathrm{b}-\mathrm{k}}=\left(V_{\mathbf{p} ; \mathbf{k}, \mathbf{k}-\mathbf{p}}^{\mathrm{b}-\mathrm{ph}}\right)^{\star}$ due to hermiticity of $H_{\mathrm{b}-\mathrm{ph}}$, and we do not specify interaction terms that result in the relaxation rates of bosons and phonons. Note that, aside from a more general momentum dependence, the boson-phonon interaction in (3) is the same as the one in the electron-phonon coupling case.

\section{BOLTZMANN APPROACH}

\section{A. Thermal drag conductivity}

Let us denote boson and phonon distribution functions as $f_{\mathbf{k}}$ and $n_{\mathbf{p}}$, respectively. In Boltzmann's approach the total heat current is the sum of the currents from each of the particle species:

$$
\mathbf{j}_{\text {tot }}=\sum_{\mathbf{k}} \mathbf{v}_{\mathbf{k}} \varepsilon_{\mathbf{k}} \delta f_{\mathbf{k}}+\sum_{\mathbf{p}} \mathbf{u}_{\mathbf{p}} \omega_{\mathbf{p}} \delta n_{\mathbf{p}}
$$

where $\mathbf{v}_{\mathbf{k}}=\partial \varepsilon_{\mathbf{k}} / \partial \mathbf{k}$ and $\mathbf{u}_{\mathbf{p}}=\partial \omega_{\mathbf{p}} / \partial \mathbf{p}$ are the velocities, the chemical potential is set to zero, and $\delta f_{\mathbf{k}}$ and $\delta n_{\mathbf{p}}$ are the non-equilibrium parts of the distribution functions of the bosons and phonons, respectively.

The distribution functions are determined from the Boltzmann equations

$$
\frac{d f_{\mathbf{k}}}{d t}=S t_{\mathbf{k}}^{\mathrm{b}}[f, n], \quad \frac{d n_{\mathbf{p}}}{d t}=S t_{\mathbf{p}}^{\mathrm{ph}}[n, f],
$$

where $S t^{\mathrm{b}(\mathrm{ph})}$ are the collision integrals which include all possible scatterings for bosons (phonons). These Boltzmann equations are coupled because the boson-phonon 
interaction in (3) yields terms in the collision integrals which depend on both $f_{\mathbf{k}}$ and $n_{\mathbf{p}}$.

Assuming the system to be in a steady state under a small uniform thermal gradient, we may linearize the Boltzmann equations in $\delta f$ and $\delta n$ to find

$$
\begin{aligned}
& \frac{\varepsilon_{\mathbf{k}}}{T} \frac{\partial f_{\mathbf{k}}^{0}}{\partial \varepsilon}\left(\mathbf{v}_{\mathbf{k}} \cdot \nabla T\right)=-\frac{\delta f_{\mathbf{k}}}{\tau_{\mathbf{k}}^{\mathrm{b}}}-\sum_{\mathbf{p}} \frac{\delta n_{\mathbf{p}}}{\tau_{\mathbf{p}, \mathbf{k}}^{\mathrm{ph}}}, \\
& \frac{\omega_{\mathbf{p}}}{T} \frac{\partial n_{\mathbf{p}}^{0}}{\partial \omega}\left(\mathbf{u}_{\mathbf{p}} \cdot \nabla T\right)=-\frac{\delta n_{\mathbf{p}}}{\tau_{\mathbf{p}}^{\mathrm{ph}}}-\sum_{\mathbf{k}} \frac{\delta f_{\mathbf{k}}}{\tau_{\mathbf{k}, \mathbf{p}}^{\mathrm{b} \rightarrow \mathrm{ph}}},
\end{aligned}
$$

where $f_{\mathbf{k}}^{0}=\left[e^{\varepsilon_{\mathbf{k}} / T}-1\right]^{-1}$ and $n_{\mathbf{p}}^{0}=\left[e^{\omega_{\mathbf{p}} / T}-1\right]^{-1}$ are the equilibrium distribution functions and the collision integrals in the right-hand sides are expanded in $\delta f$ and $\delta n$ and are considered in the relaxation-time approximation. The first terms on the right-hand sides of (6) and (77) are the usual diffusion terms, with $1 / \tau_{\mathbf{k}}^{\mathrm{b}}$ and $1 / \tau_{\mathbf{p}}^{\mathrm{ph}}$ being the transport relaxation rates of the bosons and phonons due to all possible relaxation mechanisms, as discussed in Sec. II. The second term on the right-hand side of the Boltzmann equation (6) for bosons with the momentum $\mathbf{k}$ is from the expansion of the collision integral $S t_{\mathbf{k}}^{\mathrm{b}}[f, n]$ in the phonon non-equilibrium distribution $\delta n_{\mathbf{p}}$. Because of that it contains an integral over the phonon momentum. The same is true for the phonon Boltzmann equation (7). These latter terms arise solely due to the boson-phonon coupling (3) and are due to the non-equilibrium components of the particles of opposite species. Therefore, it is natural to identify them with the drag from one species of excitations onto the other. Below we are going to explicate the relation of $1 / \tau_{\mathbf{p}, \mathbf{k}}^{\mathrm{ph} \rightarrow \mathrm{b}}$ and $1 / \tau_{\mathbf{k}, \mathbf{p}}^{\mathrm{b} \rightarrow \mathrm{ph}}$ with $V_{\mathbf{p} ; \mathbf{k}, \mathbf{k}-\mathbf{p}}^{\mathrm{b}-\mathrm{ph}}$ through the boson-phonon collision integral, but at this stage we simply use them as a short-hand notations for the "drag rates" 37

In general, Eqs. (6) and (7) reduce to integral equations for $\delta f$ and $\delta n$. However, we assume that the drag terms in (6) and (7) are small compared to the diffusion contribution, i.e., the drag rates $1 / \tau^{\mathrm{ph} \rightarrow \mathrm{b}}$ and $1 / \tau^{\mathrm{b} \rightarrow \mathrm{ph}}$ are small compared to the intrinsic boson and phonon rates $1 / \tau^{\mathrm{b}}$ and $1 / \tau^{\mathrm{ph}}$. This is equivalent to treating the boson-phonon coupling $V^{\mathrm{b}-\mathrm{ph}}$ as a perturbation. In turn, one can solve (6) and (7) iteratively by using the diffusion-only components in the integrals containing $\delta n$ and $\delta f$. This corresponds to neglecting the terms of or$\operatorname{der}\left|V^{\mathrm{b}-\mathrm{ph}}\right|^{4}$ and higher. Within this approximation, the total current in (4) is

$$
\mathbf{j}_{\text {tot }}=\mathbf{j}_{\mathrm{b}}+\mathbf{j}_{\mathrm{ph}}+\mathbf{j}_{\mathrm{d}, \mathrm{ph} \rightarrow \mathrm{b}}+\mathbf{j}_{\mathrm{d}, \mathrm{b} \rightarrow \mathrm{ph}},
$$

where $\mathbf{j}_{\mathrm{b}}$ and $\mathbf{j}_{\mathrm{ph}}$ are the usual, "diagonal" terms, and $\mathbf{j}_{\mathrm{d}, \mathrm{ph} \rightarrow \mathrm{b}}$ and $\mathbf{j}_{\mathrm{d}, \mathrm{b} \rightarrow \mathrm{ph}}$ are the currents due to the drag of phonons on bosons and vice versa. The total drag current can be written as:

$$
\begin{aligned}
j_{\mathrm{drag}}^{\alpha}= & j_{\mathrm{d}, \mathrm{ph} \rightarrow \mathrm{b}}^{\alpha}+j_{\mathrm{d}, \mathrm{b} \rightarrow \mathrm{ph}}^{\alpha}=-\frac{1}{T} \sum_{\mathbf{k}, \mathbf{p}} \tau_{\mathbf{k}}^{\mathrm{b}} \tau_{\mathbf{p}}^{\mathrm{ph}} \varepsilon_{\mathbf{k}} \omega_{\mathbf{p}} \\
& \times\left[\frac{\partial n_{\mathbf{p}}^{0}}{\partial \omega} \frac{v_{\mathbf{k}}^{\alpha} u_{\mathbf{p}}^{\beta}}{\tau_{\mathbf{p}, \mathbf{k}}^{\mathrm{ph}}}+\frac{\partial f_{\mathbf{k}}^{0}}{\partial \varepsilon} \frac{u_{\mathbf{p}}^{\alpha} v_{\mathbf{k}}^{\beta}}{\tau_{\mathbf{k}, \mathbf{p}}^{\mathrm{b} \rightarrow \mathrm{ph}}}\right] \nabla_{\beta} T
\end{aligned}
$$

where $\alpha$ and $\beta$ are vector components. Choosing the temperature gradient in the $x$-direction and assuming the conductivity tensor to be diagonal, we obtain the drag thermal conductivity:

$$
\begin{aligned}
\kappa_{\text {drag }}=\frac{1}{T} \sum_{\mathbf{k}, \mathbf{p}} & \left(v_{\mathbf{k}}^{x} \varepsilon_{\mathbf{k}} \tau_{\mathbf{k}}^{\mathrm{b}}\right)\left(u_{\mathbf{p}}^{x} \omega_{\mathbf{p}} \tau_{\mathbf{p}}^{\mathrm{ph}}\right) \\
\times & {\left[\frac{\partial n_{\mathbf{p}}^{0}}{\partial \omega} \frac{1}{\tau_{\mathbf{p}, \mathbf{k}}^{\mathrm{ph} \rightarrow \mathrm{b}}}+\frac{\partial f_{\mathbf{k}}^{0}}{\partial \varepsilon} \frac{1}{\tau_{\mathbf{k}, \mathbf{p}}^{\mathrm{b} \rightarrow \mathrm{ph}}}\right], }
\end{aligned}
$$

The above analysis thus far has been independent of the microscopic form of the boson-phonon coupling.

\section{B. Microscopic consideration.}

The "drag rates" $1 / \tau_{\mathbf{p}, \mathbf{k}}^{\mathrm{ph} \rightarrow \mathrm{b}}$ and $1 / \tau_{\mathbf{k}, \mathbf{p}}^{\mathrm{b} \rightarrow \mathrm{ph}}$ are obtained by taking variations of $f$ and $n$ in the corresponding functionals $S t[f, n]$. We now detail the derivation of one of them. The collision integral for phonons scattered off bosons via Eq. (3) contains two terms: the first one increases the number of phonons with momentum $\mathbf{p}$, the second one reduces it. They can be grouped together as:

$$
\begin{aligned}
S t_{\mathbf{p}}^{\mathrm{ph}}[n, f]= & 2 \pi \sum_{\mathbf{k}}\left|V_{\mathbf{p} ; \mathbf{k}, \mathbf{k}-\mathbf{p}}^{\mathrm{b}-\mathbf{p h}}\right|^{2} \cdot \delta\left(\varepsilon_{\mathbf{k}}-\varepsilon_{\mathbf{k}-\mathbf{p}}-\omega_{\mathbf{p}}\right) \\
& \times\left[f_{\mathbf{k}}\left(f_{\mathbf{k}-\mathbf{p}}+n_{\mathbf{p}}+1\right)-f_{\mathbf{k}-\mathbf{p}} n_{\mathbf{p}}\right]
\end{aligned}
$$

This is the complete expression of the phonon collision integral due to phonon-boson interaction (3). The subsequent linearization of (11) uses the condition $S t_{\mathbf{p}}^{\mathrm{ph}}\left[f^{0}, n^{0}\right] \equiv 0$. Writing $f=f^{0}+\delta f$ and $n=n^{0}+\delta n$ and neglecting terms of order $\delta f \delta n$ and $(\delta f)^{2}$ yields the first and second terms in the right-hand side of Eq. (7). The first one is the diffusion term while the second one is the drag term. In the latter, for the terms containing $\delta f_{\mathbf{k}-\mathbf{p}}$, we shift summation over $\mathbf{k} \rightarrow \mathbf{k}+\mathbf{p}$ so that $\delta f_{\mathbf{k}-\mathbf{p}} \rightarrow \delta f_{\mathbf{k}}$. After these manipulations, the drag rate of bosons on phonons is given by

$$
\begin{aligned}
& S t_{\mathbf{p}}^{\mathrm{ph}}\left[n^{0}, f^{0}+\delta f\right] \approx-\sum_{\mathbf{k}} \frac{\delta f_{\mathbf{k}}}{\tau_{\mathbf{k}, \mathbf{p}}^{\mathrm{b}-\mathrm{ph}}}, \\
& \frac{1}{\tau_{\mathbf{k}, \mathbf{p}}^{\mathrm{b} \rightarrow \mathrm{ph}}}=-2 \pi\left(\left|V_{\mathbf{p} ; \mathbf{k}, \mathbf{k}-\mathbf{p}}^{\mathrm{b}-\mathrm{ph}}\right|^{2}\left[f_{\mathbf{k}-\mathbf{p}}^{0}+n_{\mathbf{p}}^{0}+1\right]\right. \\
& \times \delta\left(\varepsilon_{\mathbf{k}}-\varepsilon_{\mathbf{k}-\mathbf{p}}-\omega_{\mathbf{p}}\right) \\
& \left.+\left|V_{\mathbf{p} ; \mathbf{k}+\mathbf{p}, \mathbf{k}}^{\mathrm{b}-\mathbf{p h}}\right|^{2}\left[f_{\mathbf{k}+\mathbf{p}}^{0}-n_{\mathbf{p}}^{0}\right] \cdot \delta\left(\varepsilon_{\mathbf{k}}-\varepsilon_{\mathbf{k}+\mathbf{p}}+\omega_{\mathbf{p}}\right)\right) .
\end{aligned}
$$

Note that under $\mathbf{p} \rightarrow-\mathbf{p}$, the second term in (12) changes to $\left|V_{-\mathbf{p} ; \mathbf{k}-\mathbf{p}, \mathbf{k}}^{\mathrm{b}}\right|^{2}=\left|V_{\mathbf{p} ; \mathbf{k}, \mathbf{k}-\mathbf{p}}^{\mathrm{b}-\mathbf{p h}}\right|^{2}$ and the phonon velocity changes its sign, $\mathbf{u}_{\mathbf{p}} \rightarrow-\mathbf{u}_{-\mathbf{p}}$, see discussion after (3). Using this symmetry we obtain a compact form for the component of the thermal conductivity due to the 
drag of bosons on phonons:

$$
\begin{aligned}
\kappa_{\mathrm{drag}}^{\mathrm{b} \rightarrow \mathrm{ph}} & =-\frac{2 \pi}{T} \sum_{\mathbf{k}, \mathbf{p}}\left(v_{\mathbf{k}}^{x} \varepsilon_{\mathbf{k}} \tau_{\mathbf{k}}^{\mathrm{b}}\right)\left(u_{\mathbf{p}}^{x} \omega_{\mathbf{p}} \tau_{\mathbf{p}}^{\mathrm{ph}}\right)\left|V_{\mathbf{p} ; \mathbf{k}, \mathbf{k}-\mathbf{p}}^{\mathrm{b}-\mathrm{ph}}\right|^{2} \\
\times & {\left[\frac{\partial f_{\mathbf{k}}^{0}}{\partial \varepsilon}\left[f_{\mathbf{k}-\mathbf{p}}^{0}+n_{\mathbf{p}}^{0}+1\right] \delta\left(\varepsilon_{\mathbf{k}}-\varepsilon_{\mathbf{k}-\mathbf{p}}-\omega_{\mathbf{p}}\right)(13\right.} \\
& \left.-\frac{\partial f_{\mathbf{k}}^{0}}{\partial \varepsilon}\left[f_{\mathbf{k}-\mathbf{p}}^{0}-n_{\mathbf{p}}^{0}\right] \delta\left(\varepsilon_{\mathbf{k}}-\varepsilon_{\mathbf{k}-\mathbf{p}}+\omega_{\mathbf{p}}\right)\right]
\end{aligned}
$$

The derivation of the drag rate $1 / \tau_{\mathbf{p}, \mathbf{k}}^{\mathrm{ph} \rightarrow \mathrm{b}}$ and of the drag conductivity of phonon on bosons follows similar reasoning and is presented in Appendix $\mathrm{A}$ 1. After some algebra one arrives at the following statement:

$$
\kappa_{\text {drag }}^{\mathrm{b} \rightarrow \mathrm{ph}} \equiv \kappa_{\text {drag }}^{\mathrm{ph} \rightarrow \mathrm{b}}
$$

This relation bears a simple and general physical meaning: within linear response, the non-equilibrium component of one species of particles causes the same drag on the other species as the non-equilibrium component of the other causes on the first one. That is, phonons drag bosons the same as bosons drag phonons. Thus, the total contribution to the conductivity is simply twice the contribution in Eq. (13). In addition to the algebra above, to obtain (14) we have used the following identities between the combinations of the bosonic distribution functions and their derivatives,

$$
\begin{gathered}
\left.\frac{\partial n_{\mathbf{p}}^{0}}{\partial \omega}\left[f_{\mathbf{k}-\mathbf{p}}^{0}-f_{\mathbf{k}}^{0}\right] \equiv \frac{\partial f_{\mathbf{k}}^{0}}{\partial \varepsilon}\left[f_{\mathbf{k}-\mathbf{p}}^{0}+n_{\mathbf{p}}^{0}+1\right]\right|_{\varepsilon_{\mathbf{k}}-\varepsilon_{\mathbf{k}-\mathbf{p}}=\omega_{\mathbf{p}}} \\
\left.\frac{\partial n_{\mathbf{p}}^{0}}{\partial \omega}\left[f_{\mathbf{k}-\mathbf{p}}^{0}-f_{\mathbf{k}}^{0}\right] \equiv \frac{\partial f_{\mathbf{k}}^{0}}{\partial \varepsilon}\left[f_{\mathbf{k}-\mathbf{p}}^{0}-n_{\mathbf{p}}^{0}\right]\right|_{\varepsilon_{\mathbf{k}-\mathbf{p}}-\varepsilon_{\mathbf{k}}=\omega_{\mathbf{p}}}
\end{gathered}
$$

which can be obtained with the help of $S t_{\mathbf{p}}^{\mathrm{ph}}\left[f^{0}, n^{0}\right] \equiv 0$.

Thus, within the Boltzmann formalism, the total drag thermal conductivity, to leading order in the bosonphonon coupling, is given by

$$
\begin{aligned}
\kappa_{\mathrm{drag}}= & -\frac{4 \pi}{T} \sum_{\mathbf{k}, \mathbf{p}}\left(v_{\mathbf{k}}^{x} \varepsilon_{\mathbf{k}} \tau_{\mathbf{k}}^{\mathrm{b}}\right)\left(u_{\mathbf{p}}^{x} \omega_{\mathbf{p}} \tau_{\mathbf{p}}^{\mathrm{ph}}\right)\left|V_{\mathbf{p} ; \mathbf{k}, \mathbf{k}-\mathbf{p}}^{\mathrm{b}-\mathbf{p h}}\right|^{2} \\
& \times\left[\frac{\partial f_{\mathbf{k}}^{0}}{\partial \varepsilon}\left[f_{\mathbf{k}-\mathbf{p}}^{0}+n_{\mathbf{p}}^{0}+1\right] \delta\left(\varepsilon_{\mathbf{k}}-\varepsilon_{\mathbf{k}-\mathbf{p}}-\omega_{\mathbf{p}}\right)(17\right. \\
& \left.-\frac{\partial f_{\mathbf{k}}^{0}}{\partial \varepsilon}\left[f_{\mathbf{k}-\mathbf{p}}^{0}-n_{\mathbf{p}}^{0}\right] \delta\left(\varepsilon_{\mathbf{k}}-\varepsilon_{\mathbf{k}-\mathbf{p}}+\omega_{\mathbf{p}}\right)\right]
\end{aligned}
$$

This expression is the main result of this work. We would like to note, that within the approximations discussed above this result is valid for any type of scattering, impurity, boundary or Umklapp, all being implicitly incorporated in the transport relaxation times of phonons and bosons. This expression also contains normal as well as the Umklapp boson-phonon scattering. That is, the quasimomenta in (17) are defined up to the reciprocal lattice vectors and the summation over the latter is assumed as usual. 38

\section{KUBO APPROACH}

A different theoretical approach to transport, alternative to the Boltzmann equation, is the Kubo linearresponse formalism. The great advantage of this approach is its conceptual clarity with regard to the definition of the drag thermal conductivity. It is also very effective in classifying terms by their respective order in the coupling constant as it contains them explicitly.

In Kubo's approach the uniform part of the thermal conductivity is obtained by taking the DC-limit of the imaginary part of the dynamical heat-current susceptibility $\chi^{\mu \nu}: 15$

$$
\kappa^{\mu \nu}=-\lim _{\omega \rightarrow 0} \frac{\beta}{\omega+i 0} \operatorname{Im}\left[\chi^{\mu \nu}(0, \omega+i 0)\right],
$$

where $\mu$ and $\nu$ are the spatial directions and the susceptibility is a sum of diagonal and off-diagonal terms,

$$
\chi^{\mu \nu}=\sum_{i, j=1,2} \chi_{i, j}^{\mu \nu}
$$

with the components

$$
\chi_{i, j}^{\mu \nu}(\mathbf{q}, \omega+i 0)=i \int_{0}^{\infty}\left\langle\left[j_{\mathbf{q} i}^{\mu}(t), j_{-\mathbf{q} j}^{\nu}\right]\right\rangle e^{i(\omega+i 0) t} d t,
$$

which contain the heat-currents $j_{\mathbf{q} i}^{\mu}$. In this study, the long wavelength limit of the thermal current of bosons is given by

$$
\mathbf{j}_{\mathbf{q} 1}=\sum_{\mathbf{k}} \varepsilon_{\mathbf{k}} v_{\mathbf{k}} b_{\mathbf{k}+\mathbf{q}}^{\dagger} b_{\mathbf{k}}
$$

and the phonon one by

$$
\mathbf{j}_{\mathbf{q} 2}=\sum_{\mathbf{p}} \omega_{\mathbf{p}} u_{\mathbf{p}} a_{\mathbf{p}+\mathbf{q}}^{\dagger} a_{\mathbf{p}}
$$

where the energies and velocities were defined previously in (2) and (4). For the remainder of the paper the usual limit of $\mathbf{q}=0$ is implied for the currents, however $\mathbf{q}$ is kept visible for clarity. Note that Eq. (18) is derived from the linear response to the temperature gradient, which couples to the total energy density. Therefore, apart from the bare heat currents of (21) and (22), the interaction term in the Hamiltonian (3) will also give rise to a contribution to the thermal current. This current, labeled by $\mathbf{j}_{3, \mathbf{q}}$, follows from the continuity equation,

$$
\mathbf{q} \cdot \mathbf{j}_{3, \mathbf{q}}=\left[H, H_{\mathbf{q}}\right]-\mathbf{q} \cdot\left(\mathbf{j}_{1, \mathbf{q}}+\mathbf{j}_{2, \mathbf{q}}\right),
$$

where $H_{\mathbf{q}}=\sum_{\mathbf{r}} e^{-i \mathbf{q} \cdot \mathbf{r}} H_{\mathbf{r}}$ is the Fourier transform of a position-dependent Hamiltonian energy density, $H=$ $\sum_{\mathbf{r}} H_{\mathbf{r}}$. However, $\mathbf{j}_{3, \mathbf{q}}$ does not constitute a contribution to thermal drag and we will not consider the corresponding terms in this work.

We would like to emphasize that (18) refers only to the DC-limit and does not incorporate the Drude weight. The latter is assumed to be zero henceforth. 

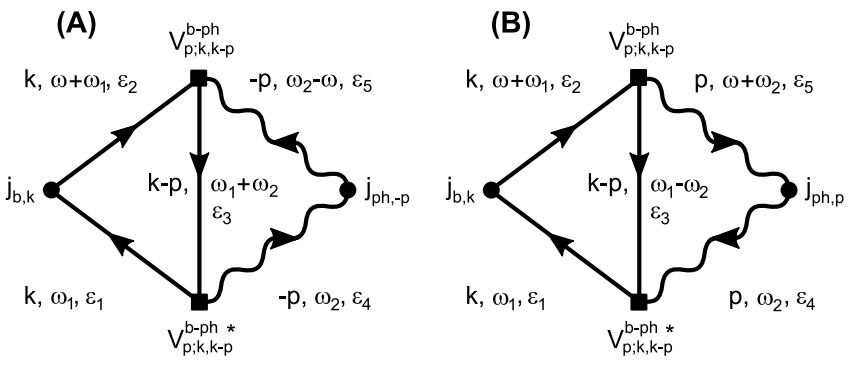

FIG. 1: Graphical representation of the lowest order, offdiagonal current-current correlations contributing to bosonphonon drag conductivity. Solid lines are bosons, wavy lines are phonons. $\varepsilon$ 's are the auxiliary frequencies used in the spectral representation approach.

The diagonal $(i=j)$ components in (20) are the "usual" diffusion terms and they do not contribute to the drag. Naturally, the drag is given by the off-diagonal currentcurrent correlation functions, $\chi_{1,2}$ and $\chi_{2,1}$. Considering the boson-phonon coupling $V_{\mathbf{p} ; \mathbf{k}, \mathbf{k}-\mathbf{p}}^{\mathrm{b}-\mathrm{ph}}$ in (3) as a perturbation, the lowest-order diagrams contributing to the drag are shown in Fig. 1 These two diagrams are the only "drag" diagrams of the order $\left|V^{\mathrm{b}-\mathrm{ph}}\right|^{2}$ that contribute to $\chi_{1,2}$. The mirror-reflection of these diagrams with respect to a vertical line yields equivalent contributions to $\chi_{2,1}$. This is, again, a graphical way of stating that in the linear response the drag of phonons on bosons and the one from bosons on phonons are identical. Thus, the total drag conductivity is given by twice the value of the diagrams in Fig. 1] The choice of the momenta in Fig. 1 is made to keep all the momentum-dependent functions between the two diagrams, such as energies and vertices, the same. Since in the diagram $A$ the phonon momentum is $\mathbf{- p}$, its sign is opposite due to the velocity in the current vertex. The analytical expression for the total drag thermal conductivity given by Fig. 1 and its mirror-reflection is:

$$
\begin{array}{r}
\kappa_{\text {drag }}=\lim _{\omega \rightarrow 0}\left[\frac{2}{T \omega} \sum_{\mathbf{k}, \mathbf{p}}\left(v_{\mathbf{k}}^{x} \varepsilon_{\mathbf{k}}\right)\left(u_{\mathbf{p}}^{x} \omega_{\mathbf{p}}\right)\left|V_{\mathbf{p} ; \mathbf{k}, \mathbf{k}-\mathbf{p}}^{\mathrm{b}-\mathrm{ph}}\right|^{2}\right. \\
\left.\operatorname{Im}\left(\Pi_{A}(\mathbf{k}, \mathbf{p}, \omega+i 0)-\Pi_{B}(\mathbf{k}, \mathbf{p}, \omega+i 0)\right)\right],
\end{array}
$$

with

$$
\begin{aligned}
\Pi_{A}(\mathbf{k}, \mathbf{p}, i \omega) & =T^{2} \sum_{\omega_{1}, \omega_{2}} G_{\mathbf{k}}\left(i \omega+i \omega_{1}\right) G_{\mathbf{k}}\left(i \omega_{1}\right) \\
& \times G_{\mathbf{k}-\mathbf{p}}\left(i \omega_{1}+i \omega_{2}\right) \bar{G}_{\mathbf{p}}\left(i \omega_{2}-i \omega\right) \bar{G}_{\mathbf{p}}\left(i \omega_{2}\right),
\end{aligned}
$$

and

$$
\begin{aligned}
\Pi_{B}(\mathbf{k}, \mathbf{p}, i \omega) & =T^{2} \sum_{\omega_{1}, \omega_{2}} G_{\mathbf{k}}\left(i \omega+i \omega_{1}\right) G_{\mathbf{k}}\left(i \omega_{1}\right) \\
& \times G_{\mathbf{k}-\mathbf{p}}\left(i \omega_{1}-i \omega_{2}\right) \bar{G}_{\mathbf{p}}\left(i \omega_{2}+i \omega\right) \bar{G}_{\mathbf{p}}\left(i \omega_{2}\right),
\end{aligned}
$$

where $G_{\mathbf{k}}(i \omega)$ and $\bar{G}_{\mathbf{p}}(i \omega)$ are the boson and phonon Green's functions, respectively.
To calculate the thermal conductivity one needs to perform the frequency summations in (25) and (26). We utilize two technical approaches for that: the first uses the spectral representation for the Matsubara Green's functions and the second one uses integration along the branch cuts of the Green's functions $\stackrel{15}{\underline{15}}$ Below we elaborate on the use of the first one while the branch cut integration approach is discussed in Appendix B

\section{A. Spectral Representation Approach}

The spectral representation for the Matsubara Green's function is:

$$
G_{\mathbf{k}}\left(i \omega_{n}\right)=\int_{\varepsilon} \frac{A_{\mathbf{k}}(\varepsilon)}{i \omega_{n}-\varepsilon}
$$

where the shorthand notation $\int_{\varepsilon} \equiv \int_{-\infty}^{\infty} d \varepsilon / 2 \pi$ and the following relation of the spectral function to the retarded Green's function, $A_{\mathbf{k}}(\varepsilon)=-2 \operatorname{Im} G_{\mathbf{k}}^{R}(\varepsilon)$, are used.

Below we calculate the contributions to the thermal conductivity from the diagram $A$ in Fig. 1, those from the diagram $B$ are discussed near the end of the section. Using the spectral function representation (27), and assigning auxiliary frequencies according to the diagrams in Fig. 1, one can rewrite $\Pi_{A}$ as:

$$
\begin{aligned}
\Pi_{A}(\mathbf{k}, \mathbf{p}, i \omega)= & \int_{\varepsilon_{1}, \ldots \varepsilon_{5}} A_{\mathbf{k}}\left(\varepsilon_{1}\right) A_{\mathbf{k}}\left(\varepsilon_{2}\right) A_{\mathbf{k}-\mathbf{p}}\left(\varepsilon_{3}\right) \\
& \times \bar{A}_{\mathbf{p}}\left(\varepsilon_{4}\right) \bar{A}_{\mathbf{p}}\left(\varepsilon_{5}\right) \cdot \bar{\Pi}_{A}\left(i \omega, \varepsilon_{1 \ldots 5}\right),
\end{aligned}
$$

where $\varepsilon_{1 \ldots 5}$ stands for the five frequencies associated with each individual line in Fig. 1(A). The frequency summation over $\omega_{1}$ and $\omega_{2}$ is now accumulated in $\bar{\Pi}_{A}$, which is given by:

$$
\begin{gathered}
\bar{\Pi}_{A}\left(i \omega, \varepsilon_{1} \ldots 5\right)=T^{2} \sum_{\omega_{1}, \omega_{2}} \frac{1}{i \omega_{1}-\varepsilon_{1}} \cdot \frac{1}{i \omega_{1}+i \omega-\varepsilon_{2}} \\
\cdot \frac{1}{i \omega_{1}+i \omega_{2}-\varepsilon_{3}} \cdot \frac{1}{i \omega_{2}-\varepsilon_{4}} \cdot \frac{1}{i \omega_{2}-i \omega-\varepsilon_{5}} \cdot
\end{gathered}
$$

Performing Matsubara frequency summations in (29) we obtain:

$$
\begin{gathered}
\bar{\Pi}_{A}\left(i \omega, \varepsilon_{1 \ldots 5}\right)=\frac{1}{i \omega+\varepsilon_{1}-\varepsilon_{2}} \cdot \frac{1}{i \omega+\varepsilon_{5}-\varepsilon_{4}} \\
\quad\left[\frac{\left(n_{3}-n_{1}\right)\left(n_{3-1}-n_{5}\right)}{i \omega+\varepsilon_{5}+\varepsilon_{1}-\varepsilon_{3}}-\frac{\left(n_{3}-n_{1}\right)\left(n_{3-1}-n_{4}\right)}{\varepsilon_{4}+\varepsilon_{1}-\varepsilon_{3}}\right. \\
\left.\quad+\frac{\left(n_{3}-n_{2}\right)\left(n_{3-2}-n_{4}\right)}{-i \omega+\varepsilon_{4}+\varepsilon_{2}-\varepsilon_{3}}-\frac{\left(n_{3}-n_{2}\right)\left(n_{3-2}-n_{5}\right)}{\varepsilon_{5}+\varepsilon_{2}-\varepsilon_{3}}\right],
\end{gathered}
$$

where $n_{i} \equiv n^{0}\left(\varepsilon_{i}\right)$ are the Bose distribution functions with the corresponding energies and $n_{i-j} \equiv n^{0}\left(\varepsilon_{i}-\varepsilon_{j}\right)$.

For the uniform, DC thermal conductivity (24) we need to take the imaginary part and the $\omega \rightarrow 0$ limit of (30): $\operatorname{Im} \bar{\Pi}_{A}\left(i \omega_{n} \rightarrow \omega+i 0\right)$ at $\omega \rightarrow 0$, which splits naturally 
into four terms:

$$
\begin{aligned}
& \operatorname{Im} \bar{\Pi}_{A}(\omega)=\sum_{m=1}^{4} \operatorname{Im} \bar{\Pi}_{A}^{(m)}(\omega) \\
& =\operatorname{Im}(\mathrm{I}) \operatorname{Re}(\mathrm{II}) \operatorname{Re}([\mathrm{III}])+\operatorname{Re}(\mathrm{I}) \operatorname{Im}(\mathrm{II}) \operatorname{Re}([\mathrm{III}]) \\
& +\operatorname{Re}(\mathrm{I}) \operatorname{Re}(\mathrm{II}) \operatorname{Im}([\mathrm{III}])-\operatorname{Im}(\mathrm{I}) \operatorname{Im}(\mathrm{II}) \operatorname{Im}([\mathrm{III}]),
\end{aligned}
$$

where I, II, and [III] are the first, second, and third factors of the product in (30), respectively, and [III] includes all the terms inside the square bracket. In what follows, we refer to the four contributions to the conductivity coming from the four terms in (31) as to $\operatorname{Im} \Pi_{A}^{(m)}$, $m=1 \ldots 4$.

\section{Boltzmann terms}

Here we explicitly evaluate the leading-order contributions to the thermal drag conductivity and show that the Kubo approach yields the same answer as the one obtained using Boltzmann equation. The discussion of the other, subleading non-Boltzmann contributions is deferred to Appendix C

We would like to assert that within the spectral representation calculation, the leading contributions are given only by $\operatorname{Im} \bar{\Pi}_{A}^{(4)}$ in (31). The rest of the terms, $\operatorname{Im} \Pi_{A}^{(1)}$, $\operatorname{Im} \Pi_{A}^{(2)}$, and $\operatorname{Im} \Pi_{A}^{(3)}$, yield results that are subleading in the sense of containing higher power of $\tau^{\prime}$ 's, which is equivalent to having higher-order terms in $V^{\mathrm{b}-\mathrm{ph}}$ and other couplings.

Consider $\operatorname{Im} \bar{\Pi}_{A}^{(4)}$ term where all three factors in (30) contribute their imaginary parts,

$$
\begin{gathered}
\operatorname{Im} \bar{\Pi}_{A}^{(4)}(\omega)=\pi^{3} \delta\left(\omega+\varepsilon_{1}-\varepsilon_{2}\right) \delta\left(\omega+\varepsilon_{5}-\varepsilon_{4}\right) \\
\quad\left[\left(n_{3}-n_{1}\right)\left(n_{3-1}-n_{5}\right) \delta\left(\omega+\varepsilon_{5}+\varepsilon_{1}-\varepsilon_{3}\right)\right. \\
\left.\quad-\left(n_{3}-n_{2}\right)\left(n_{3-2}-n_{4}\right) \delta\left(\varepsilon_{4}+\varepsilon_{2}-\varepsilon_{3}-\omega\right)\right] .
\end{gathered}
$$

In the low frequency limit Eq. (32) reduces to:

$$
\begin{aligned}
\left.\frac{\operatorname{Im} \bar{\Pi}_{A}^{(4)}(\omega)}{\omega}\right|_{\omega=0}=2 \pi^{3} \delta\left(\varepsilon_{1}-\varepsilon_{2}\right) \delta\left(\varepsilon_{5}-\varepsilon_{4}\right) \\
\quad \times\left(n_{3}-n_{1}\right) \frac{\partial n_{5}}{\partial \varepsilon} \delta\left(\varepsilon_{5}+\varepsilon_{1}-\varepsilon_{3}\right) .
\end{aligned}
$$

Substituting this into (28) and performing integrations with delta-functions, we obtain the leading contribution to $\operatorname{Im}_{A}(\mathbf{k}, \mathbf{p}, \omega)$,

$$
\begin{gathered}
\left.\frac{\operatorname{Im} \Pi_{A}^{(4)}(\mathbf{k}, \mathbf{p}, \omega)}{\omega}\right|_{\omega=0}=\frac{1}{4} \int_{\varepsilon_{1}}\left(A_{\mathbf{k}}\left(\varepsilon_{1}\right)\right)^{2} \int_{\varepsilon_{5}}\left(\bar{A}_{\mathbf{p}}\left(\varepsilon_{5}\right)\right)^{2} \\
A_{\mathbf{k}-\mathbf{p}}\left(\varepsilon_{1}+\varepsilon_{5}\right)\left[n_{1+5}-n_{1}\right] \frac{\partial n_{5}}{\partial \varepsilon} .
\end{gathered}
$$

We assume that bosons and phonons are well-defined quasiparticles with frequency-independent imaginary parts of their self-energies, $r_{\mathbf{k}}$ and $s_{\mathbf{p}}$, such that $r_{\mathbf{k}}\left(s_{\mathbf{p}}\right) \ll$ $\varepsilon_{\mathbf{k}}\left(\omega_{\mathbf{p}}\right)$. They are also related to the relaxation times used in Sec. III as $r_{\mathbf{k}}^{-1}=2 \tau_{\mathbf{k}}^{\mathrm{b}}$ and $s_{\mathbf{p}}^{-1}=2 \tau_{\mathbf{p}}^{\mathrm{ph}}$, see Ref. 15 . Thus, the spectral functions of bosons and phonons can be approximated as Lorentzians:

$$
\begin{aligned}
& A_{\mathbf{k}}\left(\varepsilon_{1}\right)=\frac{2 r_{\mathbf{k}}}{\left(\varepsilon_{1}-\varepsilon_{\mathbf{k}}\right)^{2}+r_{\mathbf{k}}^{2}}, \\
& \bar{A}_{\mathbf{p}}\left(\varepsilon_{5}\right)=\frac{2 s_{\mathbf{p}}}{\left(\varepsilon_{5}-\omega_{\mathbf{p}}\right)^{2}+s_{\mathbf{p}}^{2}} .
\end{aligned}
$$

Since the spectral functions (35) and (36) are strongly peaked at $\varepsilon_{\mathbf{k}}$ and $\omega_{\mathbf{p}}$, the main contributions in the integrals in (34) are obtained at $\varepsilon_{1} \approx \varepsilon_{\mathbf{k}}$ and $\varepsilon_{5} \approx \omega_{\mathbf{p}}$. Identifying distribution functions with that of phonons and bosons via $n\left(\omega_{\mathbf{p}}\right) \equiv n_{\mathbf{p}}^{0}, n\left(\varepsilon_{\mathbf{k}}\right)=f_{\mathbf{k}}^{0}$, and $n\left(\varepsilon_{\mathbf{k}-\mathbf{p}}\right)=$ $f_{\mathbf{k}-\mathbf{p}}^{0}$, finally yields,

$$
\begin{aligned}
&\left.\frac{\operatorname{Im} \Pi_{A}(\mathbf{k}, \mathbf{p}, \omega)}{\omega}\right|_{\omega=0} \approx 2 \pi \tau_{\mathbf{k}}^{\mathrm{b}} \tau_{\mathbf{p}}^{\mathrm{ph}} \frac{\partial n_{\mathbf{p}}^{0}}{\partial \omega}\left[f_{\mathbf{k}-\mathbf{p}}^{0}-f_{\mathbf{k}}^{0}\right] \\
& \times A_{\mathbf{k}-\mathbf{p}}\left(\varepsilon_{\mathbf{k}}+\omega_{\mathbf{p}}\right) \\
& \approx 2 \pi \tau_{\mathbf{k}}^{\mathrm{b}} \tau_{\mathbf{p}}^{\mathrm{ph}} \frac{\partial n_{\mathbf{p}}^{0}}{\partial \omega}\left[f_{\mathbf{k}-\mathbf{p}}^{0}-f_{\mathbf{k}}^{0}\right] \cdot \delta\left(\varepsilon_{\mathbf{k}}+\omega_{\mathbf{p}}-\varepsilon_{\mathbf{k}-\mathbf{p}}\right)
\end{aligned}
$$

where in the last line we have approximated the Lorenzian with the delta-function and have neglected contributions from $\Pi_{A}^{(1-3)}$ terms. As we discuss in Appendix $\mathrm{C}$, both approximation are of the same order and correspond to neglecting terms that are subleading to (37).

Repeating the same consideration for the diagram $B$ in Fig. 11 gives:

$$
\begin{aligned}
\left.\frac{\operatorname{Im} \Pi_{B}(\mathbf{k}, \mathbf{p}, \omega)}{\omega}\right|_{\omega=0} \approx & 2 \pi \tau_{\mathbf{k}}^{\mathrm{b}} \tau_{\mathbf{p}}^{\mathrm{ph}} \frac{\partial n_{\mathbf{p}}^{0}}{\partial \omega}\left[f_{\mathbf{k}-\mathbf{p}}^{0}-f_{\mathbf{k}}^{0}\right] \\
& \times \delta\left(\varepsilon_{\mathbf{k}}-\omega_{\mathbf{p}}-\varepsilon_{\mathbf{k}-\mathbf{p}}\right) .
\end{aligned}
$$

Using the identities for the distribution functions in (15), (16) and substituting (37) and (38) into (24) gives the Kubo answer for the drag component of the thermal conductivity

$$
\begin{aligned}
\kappa_{\mathrm{drag}}= & -\frac{4 \pi}{T} \sum_{\mathbf{k}, \mathbf{p}}\left(v_{\mathbf{k}}^{x} \varepsilon_{\mathbf{k}} \tau_{\mathbf{k}}^{\mathrm{b}}\right)\left(u_{\mathbf{p}}^{x} \omega_{\mathbf{p}} \tau_{\mathbf{p}}^{\mathrm{ph}}\right)\left|V_{\mathbf{p} ; \mathbf{k}, \mathbf{k}-\mathbf{p}}^{\mathrm{b}-\mathbf{p h}}\right|^{2} \\
\times & {\left[\frac{\partial f_{\mathbf{k}}^{0}}{\partial \varepsilon}\left[f_{\mathbf{k}-\mathbf{p}}^{0}+n_{\mathbf{p}}^{0}+1\right] \delta\left(\varepsilon_{\mathbf{k}}-\varepsilon_{\mathbf{k}-\mathbf{p}}-\omega_{\mathbf{p}}\right)(39)\right.} \\
& \left.-\frac{\partial f_{\mathbf{k}}^{0}}{\partial \varepsilon}\left[f_{\mathbf{k}-\mathbf{p}}^{0}-n_{\mathbf{p}}^{0}\right] \delta\left(\varepsilon_{\mathbf{k}}-\varepsilon_{\mathbf{k}-\mathbf{p}}+\omega_{\mathbf{p}}\right)\right]
\end{aligned}
$$

One can see that this is identical to the Boltzmann answer in (17). We discuss in Appendix C that the contributions from the remaining terms $\operatorname{Im} \Pi_{A}^{(m)}, m=1 \ldots 3$ and corrections to (39) due to the broadening in the spectral functions are of the order $O\left(\tau^{3}\right)$ and, therefore, can be neglected. 
One can check the consistency of the drag conductivity expression in (17) and (39) with the diagonal terms in conductivity by assuming that the leading source of the relaxations defining both the spin and phonon transport relaxation times is the spin-phonon coupling in (3). Then the diagonal and the drag conductivities are all of the same order in the spin-phonon coupling: $\kappa^{\mathrm{ph}} \sim \kappa^{\mathrm{b}} \sim \kappa_{\mathrm{drag}} \propto 1 /\left|\widetilde{V}^{\mathrm{b}-\mathrm{ph}}\right|^{2}$. This also demonstrates that in the idealized case of free spin excitations coupled to dissipationless phonons via a weak coupling all conductivities should be of the same order in that coupling.

We would like to emphasize again, that the identity between Eq. (39) and Eq. (17) is rather remarkable as they are derived starting from completely different physical formulations.

\section{FERMIONS AND OTHER}

Here we generalize the analysis of this work onto two additional cases. First, we assume the same form of the coupling to phonons (3), but consider fermions instead of bosons. This scenario is not only applicable to cases where the spin algebra has been mapped onto fermions, but is also relevant to the thermal conductivity in metals and semiconductors. The second generalization extends our drag consideration on the case of anomalous bosonic terms in the boson-phonon interaction. Such terms readily exist in the interaction of phonons with magnons in the ordered antiferromagnets, as was discussed previously $\underline{\underline{36}}$ They also exist for triplet excitations in gapped, dimerized, and other phases. The following derivations are based on the Boltzmann formalism only.

\section{A. fermions}

Coupling of fermionic excitations with phonons is, generally, of the same form as given in Eq. (31). This is obviously the case for the coupling of electrons with phonons, and is also true for the $X X Z$ spin chains when spins are represented by the Jordan-Wigner fermions. The general expression for the drag current will be still given by Eq. (9), where $\varepsilon_{\mathbf{k}}$ is replaced by $\widetilde{\varepsilon}_{\mathbf{k}}=\varepsilon_{\mathbf{k}}-\mu$, the fermion energy relative to the chemical potential, and the "drag rates" $1 / \tau^{\mathrm{ph} \rightarrow \mathrm{f}}$ and $1 / \tau^{\mathrm{f} \rightarrow \mathrm{ph}}$ are determined by the corresponding collision integral involving fermions and bosons, with $f$ now representing the fermion occupation number and $f_{\mathbf{k}}^{0}=\left(\exp \left[\left(\varepsilon_{\mathbf{k}}-\mu\right) / T\right]+1\right)^{-1}$ being the equilibrium Fermi-distribution function. One obvious difference for the probabilities is that a fermion with the momentum $\mathbf{k}$ is created with the probability given by $\left(1-f_{\mathbf{k}}\right)$. The derivation for the drag conductivity follows exactly the same steps as those for the boson-phonon case considered in Section III] Useful identities for certain combinations of $f^{0}$ and $n^{0}$, analogous to the ones in (15) and (16), are listed in Appendix $₫$ 2. Taking into consideration the above differences we obtain the total drag conductivity

$$
\begin{aligned}
\kappa_{\text {drag }}= & -\frac{4 \pi}{T} \sum_{\mathbf{k}, \mathbf{p}}\left(v_{\mathbf{k}}^{x} \widetilde{\varepsilon}_{\mathbf{k}} \tau_{\mathbf{k}}^{\mathrm{f}}\right)\left(u_{\mathbf{p}}^{x} \omega_{\mathbf{p}} \tau_{\mathbf{p}}^{\mathrm{ph}}\right)\left|V_{\mathbf{p} ; \mathbf{k}, \mathbf{k}-\mathbf{p}}^{\mathrm{f}-\mathrm{ph}}\right|^{2} \\
\times & {\left[\frac{\partial f_{\mathbf{k}}^{0}}{\partial \varepsilon}\left[1-f_{\mathbf{k}-\mathbf{p}}^{0}+n_{\mathbf{p}}^{0}\right] \delta\left(\varepsilon_{\mathbf{k}}-\varepsilon_{\mathbf{k}-\mathbf{p}}-\omega_{\mathbf{p}}\right)\right.} \\
& \left.+\frac{\partial f_{\mathbf{k}}^{0}}{\partial \varepsilon}\left[f_{\mathbf{k}-\mathbf{p}}^{0}+n_{\mathbf{p}}^{0}\right] \delta\left(\varepsilon_{\mathbf{k}}-\varepsilon_{\mathbf{k}-\mathbf{p}}+\omega_{\mathbf{p}}\right)\right] .
\end{aligned}
$$

To summarize, the phonon drag conductivity for the fermionic case (40) takes the same form as for the bosonic case (17) with two modifications: (i) $f_{\mathbf{k}-\mathbf{p}}^{0} \rightarrow-f_{\mathbf{k}-\mathbf{p}}^{0}$, (ii) $\varepsilon_{\mathbf{k}} \rightarrow \widetilde{\varepsilon}_{\mathbf{k}}=\varepsilon_{\mathbf{k}}-\mu$. Note that the second change should also be made in the case of bosons if the chemical potential for them is not zero.

We note, that the fermionic case of the drag discussed here is different from the one traditionally considered in the thermoelectric phenomena. As is mentioned in Sec. If for the electron-phonon problem in metals, the thermal-only drag effect is usually neglected because of the dominance of the electronic thermal conductivity over the phonon one $\underline{\underline{1}}$ This is not the case in many lowdimensional quantum magnets $\underline{\underline{3}, 7,32}-\underline{35}$

Regarding potentially different outcomes of the drag effect for the fermionic systems (40) compared to the bosonic ones (17) [139)], we remark that the major difference may arise due to the presence of the Fermi surface in the former cases. It is known, that the "normal" and the Umklapp scatterings contribute with opposite sign to the drag conductivity, which is discussed as one of the reasons for the suppression of the Gurevich effect in metals $, 1,16,20$ Such an effect of Umklapp can be expected to be small at low temperature for the bosonic case because all of the heat carriers are at small momenta. For the fermionic case, on the other hand, the effect of the Umklapp should be present, similarly to the electronphonon case. However, since the fermionic representation of spins is restricted to $1 \mathrm{D}$, significant differences from the traditional 3D electron-phonon consideration may also occur. Any quantitative statement on whether the drag will be more substantial for magnetic excitations obeying bosonic or fermionic statistics will depend on specific model calculations, which are not the focus of this work.

\section{B. anomalous bosonic terms}

Next we consider drag contributions in the phononboson case due to anomalous terms of the kind

$$
\mathcal{H}=\sum_{\mathbf{k}, \mathbf{p}} \widetilde{V}_{\mathbf{p} ; \mathbf{k}, \mathbf{k}-\mathbf{p}}^{\mathrm{b}-\mathbf{p h}}\left(b_{-\mathbf{k}+\mathbf{p}}^{\dagger} b_{\mathbf{k}}^{\dagger} a_{\mathbf{p}}+\text { H.c. }\right) .
$$

These describe processes involving creation of two bosons from a phonon and generation of a phonon due to the annihilation of two bosons. 
With the details of the algebra provided in Appendix $\mathrm{A} 3$, here we simply state that the approach described in Sec. III yields the following result

$$
\begin{gathered}
\kappa_{\text {drag }}=-\frac{4 \pi}{T} \sum_{\mathbf{k}, \mathbf{p}}\left(v_{\mathbf{k}}^{x} \varepsilon_{\mathbf{k}} \tau_{\mathbf{k}}^{\mathrm{f}}\right)\left(u_{\mathbf{p}}^{x} \omega_{\mathbf{p}} \tau_{\mathbf{p}}^{\mathrm{ph}}\right)\left|\widetilde{V}_{\mathbf{p} ; \mathbf{k}, \mathbf{k}-\mathbf{p}}^{\mathrm{b}-\mathrm{ph}}\right|^{2} \\
\times \frac{\partial n_{\mathbf{k}}^{0}}{\partial \omega}\left[1+f_{\mathbf{k}}^{0}+f_{\mathbf{k}-\mathbf{p}}^{0}\right] \delta\left(\varepsilon_{\mathbf{k}}+\varepsilon_{\mathbf{k}-\mathbf{p}}-\omega_{\mathbf{p}}\right) .
\end{gathered}
$$

In the case when both the "normal" (3) and "anomalous" (41) boson-phonon couplings are present, the leading contribution to the drag thermal conductivity is the sum of the results in (17) and (42).

\section{QUALITATIVE ESTIMATES}

In this section we provide a qualitative discussion of various asymptotic results that can be readily inferred from Eq. (17) for several representative spin-phonon systems with the goal of estimating when drag effects can be significant and when they are not. The temperature dependence of the drag thermal conductivity is determined by two factors: scattering lifetimes and the occupation numbers of the excitations.

\section{A. Boundary-limited regime}

First, we would like to consider gapless spin excitations with linear dispersion $\varepsilon_{\mathbf{k}} \approx v|\mathbf{k}|$, coupled to acoustic $3 \mathrm{D}$ phonons, the situation relevant to a wide variety of antiferromagnets. For the low impurity concentration and at low temperatures both phonon and boson mean-free paths can be expected to be boundarylimited, the case well documented for $\mathrm{Nd}_{2} \mathrm{CuO}_{4} \cdot \frac{39}{3}$ However, the heat carrying excitations will be few in number and the drag conductivity has to go to zero at low temperatures. A straightforward algebra in (17) yields a power-law: $\kappa_{\text {drag }} \propto T^{\gamma}$, with $\gamma=2+D_{s}+m$, where $D_{s}$ is the dimensionality of the spin system and $m$ depends on the long-wavelength $\mathbf{k}$ - and $\mathbf{p}$-dependence of the spinphonon coupling $\widetilde{V}_{\mathbf{p} ; \mathbf{k}, \mathbf{k}-\mathbf{p}}^{\mathrm{b}-\mathrm{ph}}$. In the case of $D_{s}=3$ (e.g., 3D magnons) and assuming that the coupling follows the standard form $\widetilde{V}_{\mathbf{p} ; \mathbf{k}, \mathbf{k}^{\prime}}^{\mathrm{b}-\mathrm{ph}} \propto \sqrt{p k k^{\prime}}$, which corresponds to $m=3$, altogether gives $\kappa_{\text {drag }}^{3 D} \propto T^{8}$. This should be compared with the diagonal thermal conductivities in this regime $\kappa^{\mathrm{ph}} \sim \kappa^{\mathrm{b}} \propto T^{3}$. Thus, the drag effect is, generally, subleading in the considered regime.

\section{B. Gapped spin system}

In another specific example let us consider a gapped spin system at low enough temperatures so that the occupation number of spin excitations is exponentially small: $f_{\mathbf{k}} \propto e^{-\Delta / T}$, where $\Delta$ is the gap in the spectrum. In the case when the relaxation within the spin system is only due to a weak coupling to phonons whose relaxation rate is dominated by the Umklapp processes, i.e. $\tau_{\mathbf{p}}^{\mathrm{ph}} \propto$ $e^{\widetilde{\Theta}_{D} / T}$, where $\widetilde{\Theta}_{D}$ is a fraction of the Debye energy 14 our Eq. (17) naturally leads to $\kappa_{\mathrm{drag}} \propto e^{\left(\widetilde{\Theta}_{D}-\Delta\right) / T}$. This result was obtained in Ref. 12 using the memory-matrix approach.

\section{High-temperature regime and disorder effects}

Third, we consider the high-temperature limit, for either gapped or gapless spin system, when temperature is higher than both the Debye energy and the spinexcitation energy scale, $T \gg \Theta_{D}, J$. Formally this case may raise questions regarding the transition into the disordered state, however is fully analogous to the textbook consideration of the lattice thermal conductivity at $T \gg$ $\Theta_{D} \stackrel{1}{1}$ In fact. in this region quasiparticles can be considered as strongly damped. The rates of the Umklapp scattering for spin excitations and phonons are high and are proportional to the occupation numbers of a "typical" boson or phonon, thus leading to $\tau^{\mathrm{ph}} \sim \tau^{\mathrm{b}} \propto 1 / T$. The rest of the estimate in Eq. (17) is again straightforward, giving $\kappa_{\text {drag }} \propto 1 / T$. This should be compared to the diagonal conductivities in this regime, which show the same asymptotic behavior $\stackrel{40}{{ }^{\mathrm{ph}}} \sim \kappa^{\mathrm{b}} \propto 1 / T$. This consideration, combined with the low-temperature one, implies that the drag conductivity should go through a maximum at intermediate temperatures, similar to the diagonal conductivities.

When the energy scales of the phonon and spin system are well separated, as in the cuprate-based materials where $J \gg \Theta_{D}$, another asymptotic regime is possible, $\Theta_{D} \ll T \ll J$. Intuitively, the drag can be expected to diminish together with the phonon conductivity $\left(\kappa^{\mathrm{ph}} \propto 1 / T\right)$ because phonons are sufficiently equilibrated by the phonon-phonon scattering. However, the $T$-dependence of the drag also depends on the specifics of the relaxation within the spin system. Thus, no definite conclusion on the prevalent behavior of the drag conductivity in this regime can be drawn without identifying such a relaxation.

The disorder dependence of the drag can also be considered using similar qualitative reasoning. If the disorder affects both types of excitations on equal footing, so that $\kappa^{\mathrm{ph}} \sim \kappa^{\mathrm{b}} \propto 1 / n_{\text {imp }}$, where $n_{\text {imp }}$ is the impurity concentration, then the drag conductivity diminishes as $\kappa_{\text {drag }} \propto 1 /\left(n_{\mathrm{imp}}\right)^{2}$. If the disorder can be introduced selectively in one of the sub-systems without significantly affecting the other, as in the case of lattice disorder in the ladder cuprate system $\mathrm{Ca}_{9} \mathrm{La}_{5} \mathrm{Cu}_{24} \mathrm{O}_{41} \stackrel{7}{,}$ the drag conductivity will be reduced together with the diagonal conductivity of the most affected species of excitations.

Thus, intuitive conditions for maximizing the effect of drag are the simultaneous presence of significant population of spin excitations and phonons with long scattering times. Since such conditions also imply large diago- 
nal contributions of spins and phonons to the heat current, they are typically satisfied for temperatures that are low enough in comparison with either $J$ or $\Theta_{D}$ but are above the boundary-limited regime. Note that the optimal regime for the phonon drag in thermoelectric phenomenon is often quoted as $T \sim \Theta_{D} / 5.20$ Such a regime can be of relevance to the recently reported record-breaking thermal conductivity by spin excitation in a high-purity $1 \mathrm{D}$ spin-chain material $\mathrm{SrCuO}_{2}$, Ref. 35 , where a nearly ballistic propagation of spin excitations was reported.

The issue of the separation of the drag component of the thermal conductivity from the "diagonal" one may require a series of doping experiments in which disorder is introduced deliberately to suppress the conductivity of one of the species and thus diminishing the drag as well 35

\section{D. qualitative estimate of the drag}

Lastly, we would like to come back to the problem of the gapless spin excitations with linear dispersion coupled to phonons, the problem motivated by the $1 \mathrm{D}$ spinchain and 2D layered cuprates where the spin excitations are fast and the phonons are slow, $J \gg \Theta_{D}$. Analogous to similar estimates of the thermoelectric power, $\frac{1}{1}$ and without reference to a specific model, the following consideration is not intended to be entirely rigorous, but rather is aimed at deriving an upper-limit estimate of the thermal Gurevich effect.

Let us assume that the boson relaxation is due to impurities or some other extrinsic or intrinsic mechanism while phonons are dissipationless, a consideration similar to the electron-phonon drag problem. ${ }^{1}$ Such a scenario is also potentially relevant to the $1 \mathrm{D}$ spinchain materials in low- $T$ regime. Then, the spin-phonon coupling will provide both the dissipation for phonons and the drag between phonons and spin excitations. In the drag conductivity, the phonon relaxation time $\left(\tau^{\mathrm{ph}} \propto 1 /\left|\widetilde{V}^{\mathrm{b}-\mathrm{ph}}\right|^{2}\right)$ enters together with the "drag rates" $\left(1 / \tau^{\mathrm{ph} \leftrightarrow \mathrm{b}} \propto\left|\widetilde{V}^{\mathrm{b}-\mathrm{ph}}\right|^{2}\right)$. As shown in Appendix $\mathrm{A} 4$, one can demonstrate that for quasiparticles with linear dispersions and for $\mathbf{k}$-independent boson relaxation time $\tau_{\mathbf{k}}^{\mathrm{b}}=\tau^{\mathrm{b}}$ the following simplification for the drag conductivity is possible for an arbitrary form of the coupling $\widetilde{V}_{\mathbf{p} ; \mathbf{k}, \mathbf{k}-\mathbf{p}}^{\mathrm{b}-\mathrm{ph}}$ :

$$
\kappa_{\mathrm{drag}}=-\frac{u^{2} v^{2} \tau^{\mathrm{b}}}{T} \sum_{\mathbf{p}} \frac{\partial n_{\mathbf{p}}^{0}}{\partial \omega}\left(\mathbf{p}^{x}\right)^{2}=\frac{1}{3} v^{2} \tau^{\mathrm{b}} C_{\mathrm{ph}},
$$

where $u$ and $v$ are the phonon and boson velocities, and $C_{\mathrm{ph}}$ is the phonon specific heat. Since the diagonal conductivity of bosons in this case is $\kappa^{\mathrm{b}}=\frac{1}{D_{s}} v^{2} \tau^{\mathrm{b}} C_{\mathrm{b}}$, where $D_{s}$ is the dimensionality of the spin system, the ratio of the drag conductivity to the boson one is independent of the scatterings and is defined by the boson and phonon specific heats

$$
\frac{\kappa_{\mathrm{drag}}}{\kappa_{\mathrm{b}}}=\frac{D_{s}}{3} \cdot \frac{C_{\mathrm{ph}}}{C_{\mathrm{b}}} .
$$

Since the population of phonons at a given temperature can be much larger than that of bosons, the drag conductivity can significantly exceed the one by spin excitations. Similar argument is at the core of the original proposal by Gurevich for the large thermoelectric effect in metals,, 19 where the relation $\kappa_{\mathrm{drag}} / \kappa_{\mathrm{e}}=C_{\mathrm{ph}} / C_{\mathrm{e}}$ also implies the same drift velocities of phonons and electrons.

While the parallel and the similarity between the electron-phonon drag and the thermal-only drag considered in the last example are clear, they are not complete. The difference is in the presence of another diagonal conductivity term in our consideration, $\kappa^{\mathrm{ph}} \propto 1 /\left|\widetilde{V}^{\mathrm{b}-\mathrm{ph}}\right|^{2}$, which, in the limit of the small spin-phonon coupling will dominate both $\kappa_{\mathrm{drag}}$ and $\kappa_{\mathrm{b}}$. Therefore, in general, the relation (44) does not imply the equivalence of the drift velocities of phonons and spin excitations.

\section{CONCLUSION}

In this work we have considered a two-component system of phonons and spin excitations and have obtained general expression for the off-diagonal contribution to its thermal conductivity in the lowest order of the spinphonon coupling. The off-diagonal contribution to the thermal current, referred to as thermal drag, is an enhancement of the heat flux of one of the species due to the flow of another and vice-versa. We have employed two distinct approaches, the Boltzmann formalism and the Kubo approach, to derive the spin-phonon drag thermal conductivity and have established that both approaches yield identical results, Eqs. (17) and (39). In addition, we have considered contributions to drag from anomalous terms, which generally arise from the spin-phonon coupling, e.g. in the symmetry broken phases as well as in the gapped systems characterized by triplet-like excitations. While we mainly focus on the drag between phonons and bosonic spin excitations, we have also discussed the case where the spin excitation's statistics is fermionic.

To conclude, we have obtained an explicit expression for the drag conductivity in the two-component system of phonons and spin excitations under general assumptions on the nature of interaction between them. This should allow for the practical calculations of the drag effects in a number of materials.

\section{Acknowledgments}

This work has been initiated at the Kavli Institute of Theoretical Physics and part of this work has been done at the Max Planck Institute for the Physics of Complex 
Systems, and the Aspen Institute of Physics, which we would like to thank for hospitality. This work was supported by DOE under grant DE-FG02-04ER46174 (A. L. C.) and by the DFG through Grant No. BR 1084/6-1 of FOR912 (W. B.). The research at KITP was supported by the NSF under Grant No. PHY05-51164.

\section{Appendix A: Details of the Boltzmann approach}

In this Appendix we provide some further details of the Boltzmann approach to the drag discussed in Secs. III and $\mathrm{V}$

\section{Derivation of the drag rate of phonons on bosons}

Here we derive the "drag rate" of phonons on bosons $1 / \tau_{\mathbf{p}, \mathbf{k}}^{\mathrm{ph} \rightarrow \mathrm{b}}$ in Eq. (6).

While not necessary, it is nevertheless convenient to depict processes contributing to the collision integral as "probability diagrams", see Fig. 2. The collision integral for bosons scattered off phonons via interaction (3) contains four terms, see Fig. 2(a): the first two increase the number of bosons with momentum $\mathbf{k}$ the other two scatter $\mathbf{k}$-bosons into a different state. They can be grouped together by energy-conservation to yield,

$$
\begin{aligned}
& S t_{\mathbf{k}}^{\mathrm{b}}[f, n]=2 \pi \sum_{\mathbf{p}}\left|V_{\mathbf{p} ; \mathbf{k}, \mathbf{k}-\mathbf{p}}^{\mathrm{b}-\mathbf{p h}}\right|^{2} \\
&\left(\left[f_{\mathbf{k}-\mathbf{p}} n_{\mathbf{p}}\left(f_{\mathbf{k}}+1\right)-f_{\mathbf{k}}\left(n_{\mathbf{p}}+1\right)\left(f_{\mathbf{k}-\mathbf{p}}+1\right)\right](\mathrm{A} 1)\right. \\
& \times \delta\left(\varepsilon_{\mathbf{k}}-\varepsilon_{\mathbf{k}-\mathbf{p}}-\omega_{\mathbf{p}}\right) \\
&+\left[f_{\mathbf{k}-\mathbf{p}}\left(n_{-\mathbf{p}}+1\right)\left(f_{\mathbf{k}}+1\right)-f_{\mathbf{k}} n_{-\mathbf{p}}\left(f_{\mathbf{k}-\mathbf{p}}+1\right)\right] \\
&\left.\times \delta\left(\varepsilon_{\mathbf{k}}-\varepsilon_{\mathbf{k}-\mathbf{p}}+\omega_{\mathbf{p}}\right)\right) .
\end{aligned}
$$

using $V_{-\mathbf{p} ; \mathbf{k}-\mathbf{p}, \mathbf{k}}^{\mathrm{b}-\mathrm{ph}}=\left(V_{\mathbf{p} ; \mathbf{k}, \mathbf{k}-\mathbf{p}}^{\mathrm{b}-\mathrm{ph}}\right)^{\star}$ discussed after (3), writing $f=f^{0}+\delta f$ and $n=n^{0}+\delta n$ and neglecting terms of order $\delta f \delta n$ and $\delta f \delta f$ will yield the terms proportional to $\delta f$ and $\delta n$ shown in Eq. (6). Performing this procedure and using $\delta n_{-\mathbf{p}}=-\delta n_{\mathbf{p}}$ yields the drag rate of phonons on bosons:

$$
\begin{aligned}
S t_{\mathbf{k}}^{\mathrm{b}}\left[f^{0},\right. & \left.n^{0}+\delta n\right] \approx-\sum_{\mathbf{p}} \frac{\delta n_{\mathbf{p}}}{\tau_{\mathbf{p}, \mathbf{k}}^{\mathrm{ph} \rightarrow \mathrm{b}}} \\
\frac{1}{\tau_{\mathbf{p}, \mathbf{k}}^{\mathrm{ph}-\mathrm{b}}}= & -2 \pi\left|V_{\mathbf{p} ; \mathbf{k}, \mathbf{k}-\mathbf{p}}^{\mathrm{b}-\mathbf{p h}}\right|^{2} \\
& \left(\left[f_{\mathbf{k}-\mathbf{p}}^{0}-f_{\mathbf{k}}^{0}\right] \delta\left(\varepsilon_{\mathbf{k}}-\varepsilon_{\mathbf{k}-\mathbf{p}}-\omega_{\mathbf{p}}\right)\right. \\
& \left.-\left[f_{\mathbf{k}-\mathbf{p}}^{0}-f_{\mathbf{k}}^{0}\right] \delta\left(\varepsilon_{\mathbf{k}}-\varepsilon_{\mathbf{k}-\mathbf{p}}+\omega_{\mathbf{p}}\right)\right) .
\end{aligned}
$$

Substituting this into the thermal conductivity in Eq. (10) and using relations (15) and (16) yields the thermal conductivity in (14) and (17).

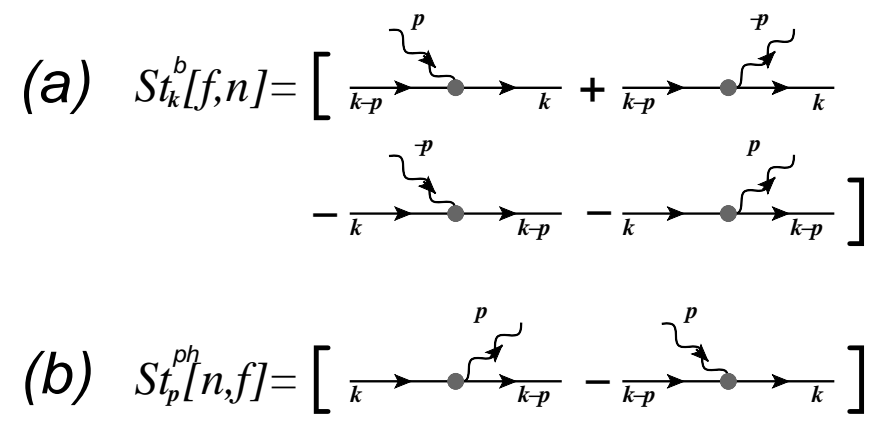

FIG. 2: Graphical representation of the collision integral terms.

The phonon collision integral discussed in Sec. IIIB is shown in Fig. 2(b).

\section{Useful identities for the fermionic case}

Identities similar to Eqs. (15) and (16) that are useful for simplifying expressions for the thermal conductivity and for relating its components to each other can also be obtained for the fermion-phonon system. They are

$$
\begin{aligned}
& \left.\frac{\partial n_{\mathbf{p}}^{0}}{\partial \omega}\left[f_{\mathbf{k}-\mathbf{p}}^{0}-f_{\mathbf{k}}^{0}\right] \equiv \frac{\partial f_{\mathbf{k}}^{0}}{\partial \varepsilon}\left[n_{\mathbf{p}}^{0}-f_{\mathbf{k}-\mathbf{p}}^{0}+1\right]\right|_{\omega_{\mathbf{p}}=\varepsilon_{\mathbf{k}}-\varepsilon_{\mathbf{k}-\mathbf{p}}} \\
& \left.\frac{\partial n_{\mathbf{p}}^{0}}{\partial \omega}\left[f_{\mathbf{k}}^{0}-f_{\mathbf{k}-\mathbf{p}}^{0}\right] \equiv \frac{\partial f_{\mathbf{k}}^{0}}{\partial \varepsilon}\left[f_{\mathbf{k}-\mathbf{p}}^{0}+n_{\mathbf{p}}^{0}\right]\right|_{\omega_{\mathbf{p}}=\varepsilon_{\mathbf{k}-\mathbf{p}}-\varepsilon_{\mathbf{k}}}
\end{aligned}
$$

These identities help to see that both contribution to the drag are equivalent.

\section{Derivation of the drag rates for the anomalous boson-phonon coupling}

The derivation of the drag in the case of the anomalous boson-phonon coupling is similar to the procedure detailed in Sec. III and Appendix $\mathrm{A}$ 1. For the coupling in (41) the scatterings describe the processes involving creation of two bosons from a phonon and generation of a phonon due to annihilation of two bosons. In that case the boson and the phonon collision integrals are described by two similar "probability diagrams", see Fig. 3.

The expression for the boson collision integral has the following form,

$$
\begin{aligned}
& S t_{\mathbf{k}}^{\mathrm{b}}[f, n]=2 \pi \sum_{\mathbf{p}}\left|\widetilde{V}_{\mathbf{p} ; \mathbf{k}, \mathbf{k}-\mathbf{p}}^{\mathrm{b}-\mathbf{p h}}\right|^{2} \cdot \delta\left(\varepsilon_{\mathbf{k}}-\varepsilon_{\mathbf{k}-\mathbf{p}}-\omega_{\mathbf{p}}\right) \\
& \quad \times\left[\left(1+f_{\mathbf{k}-\mathbf{p}}\right)\left(1+f_{\mathbf{k}}\right) n_{\mathbf{p}}-f_{\mathbf{k}-\mathbf{p}} f_{\mathbf{k}}\left(1+n_{\mathbf{p}}\right)\right] \cdot(\mathrm{A} 5)
\end{aligned}
$$

Thus, the drag rate of phonons on bosons is: 


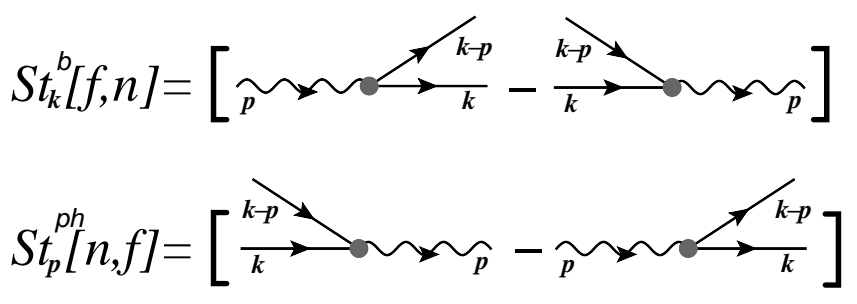

FIG. 3: Graphical representation of the collision integrals for the "anomalous" terms, Eq. (41).

$$
\begin{aligned}
& S t_{\mathbf{k}}^{\mathrm{b}}\left[f^{0}, n^{0}+\delta n\right] \approx-\sum_{\mathbf{p}} \frac{\delta n_{\mathbf{p}}}{\tau_{\mathbf{p}, \mathbf{k}}^{\mathrm{ph} \rightarrow \mathrm{b}}} \\
& \frac{1}{\tau_{\mathbf{p}, \mathbf{k}}^{\mathrm{ph} \rightarrow \mathrm{b}}}=-2 \pi\left|\widetilde{V}_{\mathbf{p} ; \mathbf{k}, \mathbf{k}-\mathbf{p}}^{\mathrm{b}-\mathbf{p h}}\right|^{2} \\
& \quad\left(1+f_{\mathbf{k}-\mathbf{p}}^{0}+f_{\mathbf{k}}^{0}\right) \delta\left(\varepsilon_{\mathbf{k}}+\varepsilon_{\mathbf{k}-\mathbf{p}}-\omega_{\mathbf{p}}\right) .
\end{aligned}
$$

Similar consideration gives the phonon collision integral:

$$
\begin{aligned}
& S t_{\mathbf{p}}^{\mathrm{ph}}[n, f]=\pi \sum_{\mathbf{k}}\left|\widetilde{V}_{\mathbf{p} ; \frac{\mathbf{k}+\mathbf{p}}{2}, \frac{\mathbf{k}-\mathbf{p}}{2}}^{\mathrm{b}-\mathbf{p h}}\right|^{2} \\
& \quad\left[\left(1+n_{\mathbf{p}}\right) f_{\frac{\mathbf{k}-\mathbf{p}}{2}} f_{\frac{\mathbf{k}+\mathbf{p}}{2}}-n_{\mathbf{p}}\left(1+f_{\frac{\mathbf{k}-\mathbf{p}}{2}}\right)\left(1+f_{\frac{\mathbf{k}+\mathbf{p}}{2}}\right)\right] \\
& \quad \times \delta\left(\varepsilon_{\frac{\mathbf{k}-\mathbf{p}}{2}}+\varepsilon_{\frac{\mathbf{k}+\mathbf{p}}{2}}-\omega_{\mathbf{p}}\right)
\end{aligned}
$$

where the factor of 2 has been removed to avoid double counting of the final states and the symmetrized notations for the momenta are used. The drag rate of bosons on phonons is given by

$$
\begin{aligned}
& S t_{\mathbf{k}}^{\mathrm{ph}}\left[n^{0}, f^{0}+\delta f\right] \approx-\sum_{\mathbf{k}} \frac{\delta f_{\mathbf{k}}}{\tau_{\mathbf{k}, \mathbf{p}}^{\mathrm{b} \rightarrow \mathrm{ph}}}, \\
& \frac{1}{\tau_{\mathbf{k}, \mathbf{p}}^{\mathrm{b} \rightarrow \mathrm{ph}}}=-2 \pi\left|\widetilde{V}_{\mathbf{p} ; \mathbf{k}, \mathbf{k}-\mathbf{p}}^{\mathrm{b}-\mathrm{ph}}\right|^{2}\left[f_{\mathbf{k}-\mathbf{p}}^{0}-n_{\mathbf{p}}^{0}\right] \\
& \times \delta\left(\varepsilon_{\mathbf{k}}+\varepsilon_{\mathbf{k}-\mathbf{p}}-\omega_{\mathbf{p}}\right) .
\end{aligned}
$$

After some algebra, the drag conductivity from bosons on phonons and phonons on bosons turn out to be identical and yield the total thermal conductivity of (42).

\section{Drag conductivity in a limiting case}

Here we derive the thermal drag conductivity under two main assumptions: the boson scattering times are independent of the momentum and those of the phonons are determined entirely by its interaction with bosons. We consider linear energy spectrum for both bosons and phonons which are given by $\varepsilon_{\mathbf{k}}=v|\mathbf{k}|$ and $\omega_{\mathbf{p}}=u|\mathbf{p}|$, respectively. Thus the drag conductivity of Eq. (17) re- duces to,

$$
\begin{aligned}
& \kappa_{\mathrm{drag}}=-\frac{4 \pi u^{2} v^{2} \tau^{\mathrm{b}}}{T} \sum_{\mathbf{k}, \mathbf{p}} \mathbf{k}^{x} \mathbf{p}^{x} \tau_{\mathbf{p}}^{\mathrm{ph}}\left|V_{\mathbf{p} ; \mathbf{k}, \mathbf{k}-\mathbf{p}}^{\mathrm{b}-\mathrm{ph}}\right|^{2} \\
& \times \frac{\partial n_{\mathbf{p}}^{0}}{\partial \omega}\left[f_{\mathbf{k}-\mathbf{p}}^{0}-f_{\mathbf{k}}^{0}\right] \\
& \times\left[\delta\left(\varepsilon_{\mathbf{k}}-\varepsilon_{\mathbf{k}-\mathbf{p}}-\omega_{\mathbf{p}}\right)-\delta\left(\varepsilon_{\mathbf{k}}-\varepsilon_{\mathbf{k}-\mathbf{p}}+\omega_{\mathbf{p}}\right)\right] .
\end{aligned}
$$

The phonon scattering time in A9 can be obtained from the phonon-boson collision integral in a standard way, similar to the derivation of the drag rates in Appendix $\mathrm{A}$. 1. Such a derivation yields,

$$
\begin{aligned}
\frac{1}{\tau_{\mathbf{p}}^{\mathrm{ph}}}= & 2 \pi \sum_{\mathbf{k}}\left|V_{\mathbf{p} ; \mathbf{k}, \mathbf{k}-\mathbf{p}}^{\mathrm{b}-\mathrm{ph}}\right|^{2} \times\left[f_{\mathbf{k}-\mathbf{p}}^{0}-f_{\mathbf{k}}^{0}\right] \\
& {\left[\delta\left(\varepsilon_{\mathbf{k}}-\varepsilon_{\mathbf{k}-\mathbf{p}}-\omega_{\mathbf{p}}\right)-\delta\left(\varepsilon_{\mathbf{k}}-\varepsilon_{\mathbf{k}-\mathbf{p}}+\omega_{\mathbf{p}}\right)\right] . }
\end{aligned}
$$

We now rewrite the drag term in a compact form,

$$
\kappa_{\mathrm{drag}}=\frac{1}{3} v^{2} \tau^{\mathrm{b}} \bar{C}_{\mathrm{ph}}
$$

using the auxiliary function $\bar{C}_{\mathrm{ph}}$, which is a "modified" phonon specific heat given by,

$$
\bar{C}_{\mathrm{ph}}=-\frac{3}{T} \sum_{\mathbf{p}} \frac{\partial n_{\mathbf{p}}^{0}}{\partial \omega}\left(u \mathbf{p}^{x}\right)^{2} F(p) .
$$

The $\mathbf{k}$-integration is now hidden in another auxiliary function $F(p)$, which is given by

$$
\begin{aligned}
& \mathbf{p}^{x} F(p)=4 \pi \tau_{\mathbf{p}}^{\mathrm{ph}} \sum_{\mathbf{k}} \mathbf{k}^{x}\left|V_{\mathbf{p} ; \mathbf{k}, \mathbf{k}-\mathbf{p}}^{\mathrm{b}-\mathrm{ph}}\right|^{2} \times\left[f_{\mathbf{k}-\mathbf{p}}^{0}-f_{\mathbf{k}}^{0}\right] \\
& {\left[\delta\left(\varepsilon_{\mathbf{k}}-\varepsilon_{\mathbf{k}-\mathbf{p}}-\omega_{\mathbf{p}}\right)-\delta\left(\varepsilon_{\mathbf{k}}-\varepsilon_{\mathbf{k}-\mathbf{p}}+\omega_{\mathbf{p}}\right)\right] .}
\end{aligned}
$$

Let us split the above expression into two terms

$$
\begin{aligned}
& I_{1}=4 \pi \tau_{\mathbf{p}}^{\mathrm{ph}} \sum_{\mathbf{k}} \mathbf{k}^{x}\left|V_{\mathbf{p} ; \mathbf{k}, \mathbf{k}-\mathbf{p}}^{\mathrm{b}-\mathrm{ph}}\right|^{2}\left[f_{\mathbf{k}-\mathbf{p}}^{0}-f_{\mathbf{k}}^{0}\right] \\
& \times \delta\left(\varepsilon_{\mathbf{k}}-\varepsilon_{\mathbf{k}-\mathbf{p}}-\omega_{\mathbf{p}}\right)
\end{aligned}
$$

and

$$
\begin{aligned}
& I_{2}=-4 \pi \tau_{\mathbf{p}}^{\mathrm{ph}} \sum_{\mathbf{k}} \mathbf{k}^{x}\left|V_{\mathbf{p} ; \mathbf{k}, \mathbf{k}-\mathbf{p}}^{\mathrm{b}-\mathrm{ph}}\right|^{2}\left[f_{\mathbf{k}-\mathbf{p}}^{0}-f_{\mathbf{k}}^{0}\right] \\
& \times \delta\left(\varepsilon_{\mathbf{k}}-\varepsilon_{\mathbf{k}-\mathbf{p}}+\omega_{\mathbf{p}}\right) .
\end{aligned}
$$

In $I_{2}$ we make the change $\mathbf{k}-\mathbf{p} \rightarrow-\mathbf{k}$,

$$
\begin{aligned}
& I_{2}=-4 \pi \tau_{\mathbf{p}}^{\mathrm{ph}} \sum_{\mathbf{k}}\left(\mathbf{k}^{x}-\mathbf{p}^{x}\right)\left|V_{\mathbf{p} ; \mathbf{p}-\mathbf{k},-\mathbf{k}}^{\mathrm{b}-\mathbf{h}}\right|^{2} \\
& \times\left[f_{\mathbf{k}-\mathbf{p}}^{0}-f_{\mathbf{k}}^{0}\right] \delta\left(\varepsilon_{\mathbf{k}}-\varepsilon_{\mathbf{k}-\mathbf{p}}-\omega_{\mathbf{p}}\right),
\end{aligned}
$$


Thus, $\bar{C}_{\mathrm{ph}}$ in $\mathrm{A12}$ is given by,

$$
\begin{aligned}
& \bar{C}_{\mathrm{ph}}=-\frac{3 u^{2}}{T} \sum_{\mathbf{p}} \frac{\partial n_{\mathbf{p}}^{0}}{\partial \omega} \mathbf{p}^{x}\left(I_{1}+I_{2}\right) \\
& =-\frac{3 u^{2}}{T} \sum_{\mathbf{p}} \frac{\partial n_{\mathbf{p}}^{0}}{\partial \omega}\left(\mathbf{p}^{x}\right)^{2}\left[4 \pi \tau_{\mathbf{p}}^{\mathrm{ph}} \sum_{\mathbf{k}}\left|V_{\mathbf{p} ; \mathbf{k}, \mathbf{k}-\mathbf{p}}^{\mathrm{b}-\mathbf{p h}}\right|^{2}\right. \\
& \left.\times\left[f_{\mathbf{k}-\mathbf{p}}^{0}-f_{\mathbf{k}}^{0}\right] \delta\left(\varepsilon_{\mathbf{k}}-\varepsilon_{\mathbf{k}-\mathbf{p}}-\omega_{\mathbf{p}}\right)\right],
\end{aligned}
$$

where we have utilized the relations $\left|V_{\mathbf{p} ; \mathbf{k}, \mathbf{k}-\mathbf{p}}^{\mathrm{b}-\mathbf{p h}}\right|^{2}=$ $\left|V_{-\mathbf{p} ; \mathbf{k}-\mathbf{p}, \mathbf{k}}^{\mathrm{b}-\mathrm{ph}}\right|^{2}$ and $\tau_{\mathbf{p}}^{\mathrm{ph}}=\tau_{-\mathbf{p}}^{\mathrm{ph}}$. Performing similar manipulations on $\tau_{\mathbf{p}}^{\mathrm{ph}}$ in (A10) one can see that the $\mathbf{k}$-integral in $\tau_{\mathbf{p}}^{\text {ph }}$ cancels exactly the one in (A17). Thus,

$$
\bar{C}_{\mathrm{ph}}=-\frac{3 u^{2}}{T} \sum_{\mathbf{p}} \frac{\partial n_{\mathbf{p}}^{0}}{\partial \omega}\left(\mathbf{p}^{x}\right)^{2} \equiv C_{\mathrm{ph}},
$$

where $C_{\mathrm{ph}}$ is the phonon specific heat. This is a rather remarkable result given the arbitrary form of boson-phonon interaction. Thus, the thermal drag conductivity expressed in terms of $\kappa_{b}=v^{2} \tau^{\mathrm{b}} C_{\mathrm{b}} / D_{s}$ is

$$
\frac{\kappa_{\mathrm{drag}}}{\kappa_{\mathrm{b}}}=\frac{D_{s}}{3} \cdot \frac{C_{\mathrm{ph}}}{C_{\mathrm{b}}}
$$

where $C_{\mathrm{b}}$ is the boson specific heat, and $D_{s}$ is the dimensionality of the spin system.

We find that $\bar{C}_{\mathrm{ph}} \equiv C_{\mathrm{ph}}$ even for the scenario when $3 \mathrm{D}$-phonons interact with $1 \mathrm{D}$ bosons. In this case, the 3D-phonon momentum can be split into $\mathbf{p}=\mathbf{p}_{\|}+\mathbf{p}_{\perp}$, the part parallel to the 1D momentum of the boson $\mathbf{k}$ and the part perpendicular to it. Thus, the boson occupation numbers in the $3 \mathrm{D}-1 \mathrm{D}$ case change to $\left(f_{\mathbf{k}-\mathbf{p}_{\|}}^{0}-f_{\mathbf{k}}^{0}\right)$ and the $\delta$-function changes to $\delta\left(\varepsilon_{\mathbf{k}}-\varepsilon_{\mathbf{k}-\mathbf{p} \|}+\omega_{\mathbf{p}}\right)$. The momentum transformation to be used for this case is $\mathbf{k}-\mathbf{p}_{\|} \rightarrow-\mathbf{k}$.

\section{Appendix B: Branch cut integration approach}

Here we derive the results of Section IV]A using a different approach, namely by converting the frequency summations in (25) and (26) to the problem of integration along the branch cuts of the Green's functions. In Section IV] A we have analyzed in detail the diagram $A$ in Fig. 1. Here we consider the derivation for the diagram $B$. Carrying out the Matsubara summation in Eq. (26), obeying the location of the branch cuts of the Green's functions and using the shorthand notation $\int_{x}=\int_{-\infty}^{+\infty} d x / 2 \pi$ leads to

$$
\begin{aligned}
& \Pi_{B}(\mathbf{k}, \mathbf{p}, i \omega)=4 \int_{x} \int_{y} n(x) n(y)[ \\
& \left\{-\operatorname{Im}\left[G_{\mathbf{k}}^{R}(y) \bar{G}_{\mathbf{p}}^{R}(x+y)\right]\right. \\
& \times G_{\mathbf{k}}(y-i \omega) \bar{G}_{\mathbf{p}}(x+y-i \omega) G_{\mathbf{k}-\mathbf{p}}^{\prime \prime}(-x) \\
& -\operatorname{Im}\left[G_{\mathbf{k}}^{R}(y) \bar{G}_{\mathbf{p}}^{R}(x+y)\right] \\
& \left.\times G_{\mathbf{k}}(y+i \omega) \bar{G}_{\mathbf{p}}(x+y+i \omega) G_{\mathbf{k}-\mathbf{p}}^{\prime \prime}(-x)\right\} \\
& +\left\{\operatorname{Im}\left[G_{\mathbf{k}}^{R}(y) G_{\mathbf{k}-\mathbf{p}}^{R}(y-x)\right]\right. \\
& \times G_{\mathbf{k}}(y-i \omega) \bar{G}_{\mathbf{p}}(x-i \omega) \bar{G}_{\mathbf{p}}^{\prime \prime}(x) \\
& +G_{\mathbf{k}}^{\prime \prime}(y) G_{\mathbf{k}}(y+i \omega) \\
& \left.\times G_{\mathbf{k}-\mathbf{p}}(y-x+i \omega) \bar{G}_{\mathbf{p}}(x-i \omega) \bar{G}_{\mathbf{p}}^{\prime \prime}(x)\right\} \\
& +\left\{G_{\mathbf{k}}^{\prime \prime}(y) G_{\mathbf{k}}(y-i \omega)\right. \\
& \times G_{\mathbf{k}-\mathbf{p}}(y-x-i \omega) \bar{G}_{\mathbf{p}}(x+i \omega) \bar{G}_{\mathbf{p}}^{\prime \prime}(x) \\
& +\operatorname{Im}\left[G_{\mathbf{k}}^{R}(y) G_{\mathbf{k}-\mathbf{p}}^{R}(y-x)\right] \\
& \left.\left.\times G_{\mathbf{k}}(y+i \omega) \bar{G}_{\mathbf{p}}(x+i \omega) \bar{G}_{\mathbf{p}}^{\prime \prime}(x)\right\}\right] \\
& =\Pi_{B}^{a}(\mathbf{k}, \mathbf{p}, i \omega)+\Pi_{B}^{b+c}(\mathbf{k}, \mathbf{p}, i \omega)
\end{aligned}
$$

where $\stackrel{(-)}{G} \underset{\mathbf{k}}{R}(x)=\stackrel{(-)}{G}\left(i \omega_{n} \rightarrow x+i 0^{+}\right)=\stackrel{(-)}{G_{\mathbf{k}}^{\prime}}(x)+i \stackrel{(-)}{G_{\mathbf{k}}^{\prime \prime}}(x)$ refers to the retarded Greens functions and their decomposition into real $\left({ }^{\prime}\right)$ and imaginary (") parts and $n(\ldots)$ is the Bose distribution function. The subscripts ' $a$ ', ' $b$ ', and ' $c$ ' refer the contributions to $\Pi_{B}(\mathbf{k}, \mathbf{p}, i \omega)$ which stem from the curly brackets labeled by Eqs. (B1), (B2), and (B3). For the DC heat conductivity we need

$$
\Pi_{B}(\mathbf{k}, \mathbf{p})=\lim _{\omega \rightarrow 0} \frac{1}{\omega} \operatorname{Im}\left[\Pi_{B}\left(\mathbf{k}, \mathbf{p}, i \omega \rightarrow \omega+i 0^{+}\right)\right] .
$$

We first take this limit focusing on $\Pi_{B}^{a}$. The variables of integration can be substituted such as to express this limit in terms of a derivative of the distribution function

$$
\begin{aligned}
\Pi_{B}^{a}(\mathbf{k}, \mathbf{p})= & 4 \int_{x} \int_{y} n(x) \frac{\partial n(y)}{\partial y} \\
& \times\left\{\operatorname{Im}\left[G_{\mathbf{k}}^{R}(y) \bar{G}_{\mathbf{p}}^{R}(x+y)\right]\right\}^{2} G_{\mathbf{k}-\mathbf{p}}^{\prime \prime}(-x) \\
\equiv & 4 \int_{x} \int_{y} n(x) \frac{\partial n(y)}{\partial y} R_{a}(x, y, \mathbf{k}, \mathbf{p}),
\end{aligned}
$$

where the abbreviation $R_{a}(x, y, \mathbf{k}, \mathbf{p})$ has been defined. A similar substitution cannot be achieved for the contributions from Eqs. (B2), and (B3). Instead, we expand 
the Greens functions to lowest order in $\omega$

$$
\begin{aligned}
\Pi_{B}^{b+c} & (\mathbf{k}, \mathbf{p})=4 \int_{x} \int_{y} n(x) n(y) \frac{\partial}{\partial \omega}[ \\
& G_{k}^{\prime \prime}(y) \bar{G}_{p}^{\prime \prime}(x) \\
\quad \times & \left\{\operatorname{Im}\left[G_{k}^{R}(y+\omega) G_{k-p}^{R}(y-x+\omega) \bar{G}_{p}^{A}(x-\omega)\right]\right. \\
& \left.-\operatorname{Im}\left[G_{k}^{R}(y-\omega) G_{k-p}^{R}(y-x-\omega) \bar{G}_{p}^{A}(x+\omega)\right]\right\} \\
& +\bar{G}_{p}^{\prime \prime}(x) \operatorname{Im}\left[G_{k}^{R}(y) G_{k-p}^{R}(y-x)\right] \\
\quad \times & \operatorname{Im}\left[G_{k}^{R}(y+\omega) \bar{G}_{p}^{R}(x+\omega)\right] \\
& \left.\left.-\operatorname{Im}\left[G_{k}^{R}(y-\omega) \bar{G}_{p}^{R}(x-\omega)\right]\right\}\right]\left.\right|_{\omega=0} \\
\equiv & 4 \int_{x} \int_{y} n(x) n(y) R_{b+c}(x, y, \mathbf{k}, \mathbf{p}),
\end{aligned}
$$

As in Eqs. (35), and (36) we introduce a phenomenological, momentum dependent one-particle self-energy for the bosons [phonons]

$$
G[\bar{G}](z, \mathbf{k})=\frac{1}{z-\varepsilon_{\mathbf{k}}\left[\omega_{\mathbf{k}}\right]+i r_{\mathbf{k}}\left[s_{\mathbf{k}}\right] \operatorname{sgn}(\operatorname{Im}(z))},
$$

where []-bracketed terms refer to phonons and $z$ is complex. Inserting this into Eq. (B6), $R_{a}(x, y, \mathbf{k}, \mathbf{p})$ turns into a rational function

$$
\begin{aligned}
R_{a}(x, y, \mathbf{k}, \mathbf{p})= & \frac{P_{a}(x, y, \mathbf{k}, \mathbf{p})}{Q_{a}(x, y, \mathbf{k}, \mathbf{p})} \\
P_{a}(x, y, \mathbf{k}, \mathbf{p}) & =-r_{\mathbf{k}-\mathbf{p}}\left[r_{\mathbf{k}} \omega_{\mathbf{p}}(x+y)+s_{\mathbf{p}} \varepsilon_{\mathbf{k}}(y)\right]^{2} \\
Q_{a}(x, y, \mathbf{k}, \mathbf{p}) & =\left[s_{\mathbf{p}}^{2}+\omega_{\mathbf{p}}(x+y)^{2}\right]^{2} \\
\times\left[r_{\mathbf{k}-\mathbf{p}}^{2}\right. & \left.+\varepsilon_{\mathbf{k}-\mathbf{p}}(-x)^{2}\right]\left[r_{\mathbf{k}}^{2}+\varepsilon_{\mathbf{k}}(y)^{2}\right]^{2},
\end{aligned}
$$

where the abbreviations $\omega_{\mathbf{k}}(x)=x-\omega_{\mathbf{k}}$ and $\varepsilon_{\mathbf{k}}(y)=y-$ $\varepsilon_{\mathbf{k}}$ are used. Note that $\omega_{\mathbf{k}}(x+y)=x+\omega_{\mathbf{k}}(y)$. Similarly, in Eq. (B7) $R_{b+c}(x, y, \mathbf{k}, \mathbf{p})$ turns into

$$
\begin{gathered}
R_{b+c}(x, y, \mathbf{k}, \mathbf{p})=\frac{P_{b+c}(x, y, \mathbf{k}, \mathbf{p})}{Q_{b+c}(x, y, \mathbf{k}, \mathbf{p})} \\
P_{b+c}(x, y, \mathbf{k}, \mathbf{p})=2 s_{\mathbf{p}}\left\{-2 r_{\mathbf{k}} r_{\mathbf{k}-\mathbf{p}}\left[\omega_{\mathbf{p}}(x)^{2}+s_{\mathbf{p}}^{2}\right]\right. \\
\quad \times\left[r_{\mathbf{k}}^{2}+\varepsilon_{\mathbf{k}}(y)^{2}\right]\left\{r_{\mathbf{k}-\mathbf{p}}\left[\omega_{\mathbf{p}}(x) r_{\mathbf{k}}-s_{\mathbf{p}} \varepsilon_{\mathbf{k}}(y)\right]-\left[r_{\mathbf{k}} s_{\mathbf{p}}\right.\right. \\
\left.\left.+\omega_{\mathbf{p}}(x) \varepsilon_{\mathbf{k}}(y)\right] \varepsilon_{\mathbf{k}-\mathbf{p}}(y-x)\right\}+\left[r_{\mathbf{k}-\mathbf{p}}^{2}+\varepsilon_{\mathbf{k}-\mathbf{p}}(y-x)^{2}\right] \\
\times\left(-\left(r_{\mathbf{k}}-r_{\mathbf{k}-\mathbf{p}}\right) s_{\mathbf{p}}\left[\omega_{\mathbf{p}}(x)^{2}+s_{\mathbf{p}}^{2}\right] \varepsilon_{\mathbf{k}}(y)\left[r_{\mathbf{k}}^{2}+\varepsilon_{\mathbf{k}}(y)^{2}\right]\right. \\
+\omega_{\mathbf{p}}(x) s_{\mathbf{p}}\left\{-2 r_{\mathbf{k}}^{4} r_{\mathbf{k}-\mathbf{p}}+r_{\mathbf{k}}^{4} s_{\mathbf{p}}-r_{\mathbf{k}}^{3} r_{\mathbf{k}-\mathbf{p}} s_{\mathbf{p}}\right. \\
+r_{\mathbf{k}}^{2} s_{\mathbf{p}} \varepsilon_{\mathbf{k}}(y)^{2}+3 r_{\mathbf{k}} r_{\mathbf{k}-\mathbf{p}} s_{\mathbf{p}} \varepsilon_{\mathbf{k}}(y)^{2}+2 r_{\mathbf{k}-\mathbf{p}} \varepsilon_{\mathbf{k}}(y)^{4} \\
\left.+4 r_{\mathbf{k}} \varepsilon_{\mathbf{k}}(y)\left[r_{\mathbf{k}}\left(r_{\mathbf{k}}+s_{\mathbf{p}}\right)+\varepsilon_{\mathbf{k}}(y)^{2}\right] \varepsilon_{\mathbf{k}-\mathbf{p}}(y-x)\right\} \\
+\omega_{\mathbf{p}}(x)^{3} r_{\mathbf{k}}\left\{r_{\mathbf{k}}^{3}-r_{\mathbf{k}}^{2} r_{\mathbf{k}-\mathbf{p}}+3 r_{\mathbf{k}-\mathbf{p}} \varepsilon_{\mathbf{k}}(y)^{2}\right. \\
\left.\left.\left.+r_{\mathbf{k}} \varepsilon_{\mathbf{k}}(y)\left[\varepsilon_{\mathbf{k}}(y)+4 \varepsilon_{\mathbf{k}-\mathbf{p}}(y-x)\right]\right\}\right)\right\} \\
Q_{b+c}(x, y, \mathbf{k}, \mathbf{p})=\left[s_{\mathbf{p}}^{2}+\omega_{\mathbf{p}}^{2}(x)\right]^{3}\left[r_{\mathbf{k}}^{2}+\varepsilon_{\mathbf{k}}^{2}(y)\right]^{3} \\
\quad \times\left[r_{\mathbf{k}-\mathbf{p}}^{2}+\varepsilon_{\mathbf{k}-\mathbf{p}}^{2}(y-x)\right]^{2}
\end{gathered}
$$

$\Pi_{B}^{a(b+c)}(\mathbf{k}, \mathbf{p})$ will be evaluated assuming, as before, that the bosons and phonons are quasiparticles with $r_{\mathbf{k}}\left(s_{\mathbf{k}}\right) \ll$ $\varepsilon_{\mathbf{k}}\left(\omega_{\mathbf{k}}\right)$. In that case, expressions valid to leading order in $r_{\mathbf{k}}\left(s_{\mathbf{k}}\right) / \varepsilon_{\mathbf{k}}\left(\omega_{\mathbf{k}}\right)$ for Eqs. (B6), and (B7) are obtained from the residues of $R_{a(b+c)}(x, y, \mathbf{k}, \mathbf{p})$ alone, while assuming the distribution functions to be holomorphic and retaining only their lowest-order non-vanishing derivatives. Moreover, any imaginary part of the arguments of the distribution functions arising in that process can be dropped. Since the poles of $R_{a(b+c)}(x, y, \mathbf{k}, \mathbf{p})$ stem from quadratic equations at most, this calculation can be done analytically. We emphasize that the proper evaluation of the higher-order contributions in $r_{\mathbf{k}}\left(s_{\mathbf{k}}\right) / \varepsilon_{\mathbf{k}}\left(\omega_{\mathbf{k}}\right)$ to $\Pi_{B}^{a(b+c)}(\mathbf{k}, \mathbf{p})$ would require a treatment of the pole structure of the Bose distribution functions and their derivatives. Analytically this is not feasible given $R_{a(b+c)}(x, y, \mathbf{k}, \mathbf{p})$. This also implies that the "non-Boltzmann" terms of Appendix C are not a systematic account of all next-leading order corrections. The leading-order analytic calculation is tedious but straightforward. After some algebra we arrive at

$$
\begin{array}{r}
\Pi_{B}^{a}(\mathbf{k}, \mathbf{p})=-\frac{1}{2 r_{\mathbf{k}} s_{\mathbf{p}}} \frac{\partial n\left(\varepsilon_{\mathbf{k}}\right)}{\partial \varepsilon_{\mathbf{k}}} n\left(-\varepsilon_{\mathbf{k}-\mathbf{p}}\right) \frac{\eta_{\mathbf{k p}}}{\left(\eta_{\mathbf{k p}}^{2}+e_{\mathbf{k p}}^{2}\right)} \\
\Pi_{B}^{b+c}(\mathbf{k}, \mathbf{p})=\frac{1}{2 r_{\mathbf{k}} s_{\mathbf{p}}} \frac{\partial n\left(\varepsilon_{\mathbf{k}}\right)}{\partial \varepsilon_{\mathbf{k}}} n\left(\omega_{\mathbf{p}}\right) \frac{\eta_{\mathbf{k p}}}{\left(\eta_{\mathbf{k p}}^{2}+e_{\mathbf{k p}}^{2}\right)},
\end{array}
$$

where $\eta_{\mathbf{k p}}=r_{\mathbf{k}}+r_{\mathbf{k}-\mathbf{p}}+s_{\mathbf{p}}$ and $e_{\mathbf{k p}}=\omega_{\mathbf{p}}-\varepsilon_{\mathbf{k}}+\varepsilon_{\mathbf{k}-\mathbf{p}}$. Thus, the rightmost fraction in both expressions can be approximated by $\pi \delta\left(\omega_{\mathbf{p}}-\varepsilon_{\mathbf{k}}+\varepsilon_{\mathbf{k}-\mathbf{p}}\right)$. The corresponding constraint $\omega_{\mathbf{p}} \approx \varepsilon_{\mathbf{k}}-\varepsilon_{\mathbf{k}-\mathbf{p}}$ has also been used to rearrange the arguments of the distribution functions in Eq. (B11). Diagram $A$ in Fig. 1 can be obtained directly from the preceding derivation by relabeling $\omega_{2} \rightarrow-\omega_{2}$ and by realizing that $-i \omega_{n}-\omega_{\mathbf{p}}+i s_{\mathbf{p}} \operatorname{sgn}\left(\operatorname{Im}\left(-i \omega_{n}\right)\right)=$ $-\left[i \omega_{n}+\omega_{\mathbf{p}}+i s_{\mathbf{p}} \operatorname{sgn}\left(\operatorname{Im}\left(i \omega_{n}\right)\right)\right]$. I.e. $\chi_{A}$ can be obtained from Eq. (B11) simply by using the symmetries: $s_{\mathbf{p}}=s_{-\mathbf{p}}, \omega_{\mathbf{p}} \rightarrow \omega_{-\mathbf{p}}, u_{\mathbf{p}}^{\mu}=-u_{-\mathbf{p}}^{\mu}$, and by replacing $\omega_{\mathbf{p}} \rightarrow-\omega_{\mathbf{p}}$. Since $\kappa^{\mu \nu}=\kappa_{A}^{\mu \nu}+\kappa_{B}^{\mu \nu}$ and the total drag is $\kappa_{\text {drag }}=\kappa_{12}+\kappa_{21}$, the final result is

$$
\begin{aligned}
& \kappa_{\text {drag }}=-\frac{4 \pi}{T} \sum_{\mathbf{k}, \mathbf{p}} v_{\mathbf{k}}^{x} \varepsilon_{\mathbf{k}} u_{\mathbf{p}}^{x} \omega_{\mathbf{p}}\left|V_{\mathbf{p} ; \mathbf{k}, \mathbf{k}-\mathbf{p}}^{\mathrm{b}-\mathrm{ph}}\right|^{2} \tau_{\mathbf{k}}^{b} \tau_{\mathbf{p}}^{p h} \times \\
&\left\{\frac{\partial f_{\mathbf{k}}^{0}}{\partial \varepsilon_{\mathbf{k}}}\left[1+f_{\mathbf{k}-\mathbf{p}}^{0}+n_{\mathbf{p}}^{0}\right] \delta\left(\varepsilon_{\mathbf{k}}-\varepsilon_{\mathbf{k}-\mathbf{p}}-\omega_{\mathbf{p}}\right)\right. \\
&\left.-\frac{\partial f_{\mathbf{k}}^{0}}{\partial \varepsilon_{\mathbf{k}}}\left[f_{\mathbf{k}-\mathbf{p}}^{0}-n_{\mathbf{p}}^{0}\right] \delta\left(\varepsilon_{\mathbf{k}}-\varepsilon_{\mathbf{k}-\mathbf{p}}+\omega_{\mathbf{p}}\right)\right\} .
\end{aligned}
$$

where we have renamed the Bose distribution functions with arguments $\varepsilon_{\mathbf{k}}\left(\omega_{\mathbf{k}}\right)$ to $f_{\mathbf{k}}^{0}\left(n_{\mathbf{k}}^{0}\right)$, as in section [IV]A. This result is identical to Eq. (39). Thus, both technical approaches within the Kubo formalism yield the same answer. 


\section{Appendix C: Non-Boltzmann contributions}

In Section IV]A we have discussed contributions from $\operatorname{Im} \bar{\Pi}_{A}^{(4)}(\omega)$ and showed that they lead to the results identical to the ones from Boltzmann theory. In the following we will discuss additional contributions to drag thermal conductivity from the remaining terms of Eq. (31), $\operatorname{Im} \bar{\Pi}_{A}^{(m)}(\omega), m=1,2$ and 3, which, however, are subleading and can be safely neglected. Evaluation of these terms is rather cumbersome, and for illustrative purposes we focus only on $\operatorname{Im} \bar{\Pi}_{A}^{(2)}(\omega)$, which is given by

$$
\begin{aligned}
\operatorname{Im} & \bar{\Pi}_{A}^{(2)}(\mathbf{k}, \mathbf{p}, \omega)=-\frac{1}{2} \int_{\varepsilon_{1}} \int_{\varepsilon_{2}} \int_{\varepsilon_{3}} \int_{\varepsilon_{5}} A_{\mathbf{k}}\left(\varepsilon_{1}\right) A_{\mathbf{k}}\left(\varepsilon_{2}\right) A_{\mathbf{k}-\mathbf{p}}\left(\varepsilon_{3}\right) \bar{A}_{\mathbf{p}}\left(\varepsilon_{5}+\omega\right) \bar{A}_{\mathbf{p}}\left(\varepsilon_{5}\right) P \frac{1}{\varepsilon_{1}-\varepsilon_{2}+\omega} \\
& P\left[\frac{\left(n_{3}-n_{1}\right)\left(n_{3-1}-n_{5}\right)}{\omega+\varepsilon_{5}+\varepsilon_{1}-\varepsilon_{3}}-\frac{\left(n_{3}-n_{1}\right)\left(n_{3-1}-n_{5+\omega}\right)}{\omega+\varepsilon_{5}+\varepsilon_{1}-\varepsilon_{3}}+\frac{\left(n_{3}-n_{2}\right)\left(n_{3-2}-n_{5+\omega}\right)}{\varepsilon_{5}+\varepsilon_{2}-\varepsilon_{3}}-\frac{\left(n_{3}-n_{2}\right)\left(n_{3-2}-n_{5}\right)}{\varepsilon_{5}+\varepsilon_{2}-\varepsilon_{3}}\right],
\end{aligned}
$$

where $P$ stands for the principal value. In the limit of zero-frequency, $\omega \rightarrow 0$, we obtain,

$$
\left.\frac{\operatorname{Im} \bar{\Pi}_{A}^{(2)}(\mathbf{k}, \mathbf{p}, \omega)}{\omega}\right|_{\omega=0}=\int_{\varepsilon_{1}} \int_{\varepsilon_{2}} \int_{\varepsilon_{3}} \int_{\varepsilon_{5}} A_{\mathbf{k}}\left(\varepsilon_{1}\right) A_{\mathbf{k}}\left(\varepsilon_{2}\right) A_{\mathbf{k}-\mathbf{p}}\left(\varepsilon_{3}\right)\left(\bar{A}_{\mathbf{p}}\left(\varepsilon_{5}\right)\right)^{2} P \frac{1}{\varepsilon_{1}-\varepsilon_{2}} P \frac{\left(n_{1}-n_{3}\right)}{\varepsilon_{1}+\varepsilon_{5}-\varepsilon_{3}} \frac{\partial n_{5}}{\partial \varepsilon_{5}}
$$

Using the spectral representation (35) one can easily perform integrations in $\varepsilon_{2}$. We further simplify the expression by performing the $\varepsilon_{1}$ and $\varepsilon_{3}$ integrations on the terms containing $n_{3}$ and $n_{1}$, respectively, to obtain

$$
\begin{aligned}
\left.\frac{\operatorname{Im} \bar{\Pi}_{A}^{(2)}(\mathbf{k}, \mathbf{p}, \omega)}{\omega}\right|_{\omega=0} & =\int_{\varepsilon_{1}} \int_{\varepsilon_{5}} A_{\mathbf{k}}\left(\varepsilon_{1}\right)\left(\bar{A}_{\mathbf{p}}\left(\varepsilon_{5}\right)\right)^{2}\left[\frac{1}{2} \frac{y_{1}}{y_{1}^{2}+r_{\mathbf{k}}^{2}} \frac{\partial n_{1}}{\partial \varepsilon_{1}}+\left(\frac{1}{2} \frac{\partial}{\partial y_{1}} \frac{y_{1}}{y_{1}^{2}+r_{\mathbf{k}}^{2}}\right) n_{1}\right] \frac{\partial n_{5}}{\partial \varepsilon_{5}} \\
& -\int_{\varepsilon_{3}} \int_{\varepsilon_{5}} A_{\mathbf{k}-\mathbf{p}}\left(\varepsilon_{3}\right)\left(\bar{A}_{\mathbf{p}}\left(\varepsilon_{5}\right)\right)^{2}\left(\frac{1}{2} \frac{\partial}{\partial y} \frac{y}{y^{2}+r_{\mathbf{k}}^{2}}\right) n_{3} \frac{\partial n_{5}}{\partial \varepsilon_{5}}
\end{aligned}
$$

where $y=\left(\varepsilon_{\mathbf{k}}+\varepsilon_{5}-\varepsilon_{3}\right)$ and $y_{1}=\left(\varepsilon_{1}+\varepsilon_{5}-\varepsilon_{\mathbf{k}-\mathbf{p}}\right)$. The contributions from all three terms in the above expression are of the same order. Consider contributions from the first term which is given by

$$
\frac{1}{2} \int_{\varepsilon_{1}} \int_{\varepsilon_{5}} A_{\mathbf{k}}\left(\varepsilon_{1}\right)\left(\bar{A}_{\mathbf{p}}\left(\varepsilon_{5}\right)\right)^{2} \frac{y_{1}}{y_{1}^{2}+r_{\mathbf{k}}^{2}} \frac{\partial n_{1}}{\partial \varepsilon_{1}} \frac{\partial n_{5}}{\partial \varepsilon_{5}} \approx \frac{\pi}{2} \frac{1}{r_{\mathbf{k}} s_{\mathbf{p}}} \frac{\partial n_{\mathbf{p}}^{0}}{\partial \omega} \frac{\partial f_{\mathbf{k}}^{0}}{\partial \varepsilon} \cdot\left(\varepsilon_{\mathbf{k}}+\omega_{\mathbf{p}}-\varepsilon_{\mathbf{k}-\mathbf{p}}\right) \cdot \delta\left(\varepsilon_{\mathbf{k}}+\omega_{\mathbf{p}}-\varepsilon_{\mathbf{k}-\mathbf{p}}\right)
$$

It appears that this expression contains the factor of the type $x \cdot \delta(x)$, which implies $x \equiv 0$. However, under strict consideration, i.e., taking into account finite lifetime $r_{\mathbf{k}}, x$ is non-zero and is of the same order as the "spread" of the $\delta$-function $\left(x \sim r_{\mathbf{k}}\right)$. From a direct comparison of Eq. (C4) with Eq. (37), we conclude that contributions from (C4) are smaller by the factor $\left(r_{\mathbf{k}}\left|\partial f_{\mathbf{k}}^{0} / \partial \varepsilon\right| / f_{\mathbf{k}}^{0}\right) \sim r_{\mathbf{k}} / \varepsilon_{\mathbf{k}} \ll 1$. Thus, the thermal conductivity contributions from (C4) and from the rest of the terms of (C3) can be neglected in comparison to the Boltzmann terms of Eq. (37). For the similar reason it is justified to use the delta-function form in Eq. (39) for the leading contributions, because the broadening in the spectral function only yields a subleading correction of higher order in $r_{\mathbf{k}}\left[s_{\mathbf{p}}\right]$.

1 J. M. Ziman, Principles of the Theory of Solids, Cambridge University Press, (1979).

2 A. A. Abrikosov, Fundamentals of the Theory of Metals, North Holland, (1988).

3 C. Hess, Eur. Phys. J. Special Topics 151, 73 (2007).

${ }^{4}$ F. Heidrich-Meisner, A. Honecker, and W. Brenig, Eur. Phys. J. Special Topics 151, 135 (2007).

5 X. Zotos, F. Naef, P. Prelovšek, Phys. Rev. B 55, 11029 (1997).

6 X. Zotos and P. Prelovsek, in Interacting Electrons in Low Dimensions, Kluwer Academic Publishers, (2003).

7 C. Hess, C. Baumann, U. Ammerahl, B. Büchner, F.
Heidrich-Meisner, W. Brenig, and A. Revcolevschi, Phys. Rev. B 64, 184305 (2001).

8 E. Shimshoni, N. Andrei, and A. Rosch, Phys. Rev. B 68, 104401 (2003).

9 A. V. Rozhkov and A. L. Chernyshev, Phys. Rev. Lett. 94, 087201 (2005); Phys. Rev. B 72, 104423 (2005).

10 K. Louis, P. Prelovsek, and X. Zotos, Phys. Rev. B 74, 235118 (2006).

11 L. E. Gurevich and G. A. Roman, Sov. Phys. Sol. State 8, 2102 (1967).

12 E. Boulat, P. Mehta, N. Andrei, E. Shimshoni, and A. Rosch, Phys. Rev. B 76, 214411 (2007). 
13 A. L. Chernyshev, J. Magn. Magn. Mater. 310, 1263 (2007).

14 R. Berman, Thermal conduction in solids, Clarendon Press, Oxford, (1976).

15 G. D. Mahan, Many-Particle Physics, Plenum Press, New York London, (1990).

${ }^{16}$ F. J. Blatt, P. A. Schroeder, C. L. Foiles, and D. Grieg, Thermoelectric Power of Metals, New York, Plenum Press, (1976), and references therein.

17 T. Holstein, Ann. Phys. 29, 410 (1964).

18 J. Bass, W. P. Pratt, and P. A. Schroeder, Rev. Mod. Phys. 62, 645 (1990).

19 L. Gurevich, Journ. of Phys. (Moscow) 9, 477 (1945); 10, 67 (1946).

20 J. M. Ziman, Electrons and phonons, Oxford, Clarendon Press, (1963).

21 J. Rammer and H. Smith, Rev. Mod. Phys. 58, 323 (1986).

22 H. Mori, Prog. Theor. Phys. (Kyoto) 33, 423 (1965); 34, 399 (1965).

${ }^{23}$ W. Götze and P. Wölfle, Phys. Rev. B 6, 1226 (1972).

24 J. S. Langer, Phys. Rev. 120, 714 (1960).

${ }^{25}$ O. V. Konstantinov and V. I. Perel, Zh. Eksperim. Theor. Fiz. 39, 197 (1960); [Sov. Phys. JETP 12, 142 (1961)].

${ }^{26}$ K. Yamada, Prog. Theor. Phys. (Kyoto) 28, 299 (1962).

${ }^{27}$ K. H. Michel and J. M. J. van Leeuwe, Physica 30, 410 (1964).
28 K. H. Michel, Physica 30, 2194 (1964).

29 A. Ron, Nuovo Cimento 34, 1494 (1964).

30 N. M. Plakida, Zh. Eksperim. Theor. Fiz. 53, 2041 (1968); [Sov. Phys. JETP 26, 115 (1968)].

31 W. Hánsch and G. D. Mahan, Phys. Rev. B 28, 1902 (1983).

32 A. V. Sologubenko, K. Giannó, H. R. Ott, A. Vietkine, and A. Revcolevschi, Phys. Rev. B 64, 054412 (2001).

33 A. V. Sologubenko, H. R. Ott, G. Dhalenne, and A. Revcolevschi, Europhys. Lett. 62, 540 (2003).

34 P. Ribeiro, C. Hess, P. Reutler, G. Roth, and B. Büchner, J. Mag. Mag. Mat. 290-291, 334 (2005).

35 N. Hlubek, P. Ribeiro, R. Saint-Martin, A. Revcolevschi, G. Roth, G. Behr, B. Büchner, and C. Hess, Phys. Rev. B 81, 020405(R) (2010).

36 G. S. Dixon, Phys. Rev. B 21, 2851 (1980).

37 C. Herring, Phys. Rev. 96, 1163 (1954).

38 E. M. Lifshitz and L. P. Pitaevskii, Physical Kinetics, (Pergamon Press, Oxford, 1981).

39 S. Y. Li, J.-B. Bonnemaison, A. Payeur, P. Fournier, C. H. Wang, X. H. Chen, and Louis Taillefer, Phys. Rev. B 77, 134501 (2008).

40 This result follows from $\kappa^{\mathrm{ph}(\mathrm{b})} \sim u^{2}\left(v^{2}\right) C_{\mathrm{ph}(\mathrm{b})} \tau^{\mathrm{ph}(\mathrm{b})}$, where the specific heat $C_{\mathrm{ph}(\mathrm{b})} \approx$ const at high temperatures. 